\title{
HAT-P-34b-HAT-P-37b: FOUR TRANSITING PLANETS MORE MASSIVE THAN JUPITER ORBITING MODERATELY BRIGHT STARS*
}

\author{
G. Á. Bakos ${ }^{1,2,12}$, J. D. Hartman ${ }^{1,2}$, G. Torres $^{2}$, B. Béky ${ }^{2}$, D. W. Latham ${ }^{2}$, L. A. Buchhave ${ }^{3}$, \\ Z. Csubry ${ }^{1,2}$, G. Kovács ${ }^{4}$, A. Bieryla ${ }^{2}$, S. Quinn ${ }^{2}$, T. SzKLenÁR ${ }^{2}$, G. A. EsQuerdo ${ }^{2}$, A. ShPorer ${ }^{5,6}$,

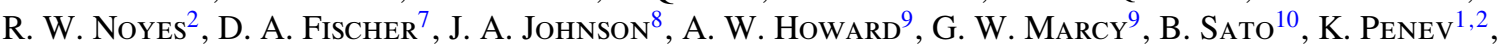 \\ M. EveretT ${ }^{2}$, D. D. SASSElov ${ }^{2}$, G. FÛ́RÉSZ ${ }^{2}$, R. P. StefaniK ${ }^{2}$, J. LÁZÁR ${ }^{11}$, I. PAPP ${ }^{11}$, AND P. SÁRI ${ }^{11}$ \\ ${ }^{1}$ Department of Astrophysical Sciences, Princeton University, Princeton, NJ 08544, USA; gbakos@ astro.princeton.edu \\ ${ }^{2}$ Harvard-Smithsonian Center for Astrophysics, Cambridge, MA, USA \\ ${ }^{3}$ Niels Bohr Institute, University of Copenhagen, DK-2100, Denmark, and Centre for Star and Planet Formation, \\ Natural History Museum of Denmark, DK-1350 Copenhagen \\ ${ }^{4}$ Konkoly Observatory, Budapest, Hungary \\ ${ }^{5}$ LCOGT, 6740 Cortona Drive, Santa Barbara, CA, USA \\ ${ }^{6}$ Department of Physics, Broida Hall, UC Santa Barbara, CA, USA \\ ${ }^{7}$ Astronomy Department, Yale University, New Haven, CT, USA \\ ${ }^{8}$ California Institute of Technology, Department of Astrophysics, MC 249-17, Pasadena, CA, USA \\ ${ }^{9}$ Department of Astronomy, University of California, Berkeley, CA, USA \\ ${ }^{10}$ Department of Earth and Planetary Sciences, Tokyo Institute of Technology, 2-12-1 Ookayama, Meguro-ku, Tokyo 152-8551, Japan \\ ${ }^{11}$ Hungarian Astronomical Association, Budapest, Hungary \\ Received 2011 December 23; accepted 2012 April 13; published 2012 June 11
}

\begin{abstract}
We report the discovery of four transiting extrasolar planets (HAT-P-34b-HAT-P-37b) with masses ranging from 1.05 to $3.33 M_{\mathrm{J}}$ and periods from 1.33 to 5.45 days. These planets orbit relatively bright $\mathrm{F}$ and $\mathrm{G}$ dwarf stars (from $V=10.16$ to $V=13.2)$. Of particular interest is HAT-P-34b which is moderately massive $\left(3.33 M_{\mathrm{J}}\right)$, has a high eccentricity of $e=0.441 \pm 0.032$ at a period of $P=5.452654 \pm 0.000016$ days, and shows hints of an outer component. The other three planets have properties that are typical of hot Jupiters.
\end{abstract}

Key words: planetary systems - stars: individual (HAT-P-34, GSC 1622-01261, HAT-P-35, GSC 0203-01079, HAT-P-36,......) - techniques: photometric - techniques: spectroscopic

Online-only material: color figures, machine-readable and VO tables

\section{INTRODUCTION}

Transiting extrasolar planets (TEPs) provide unique opportunities to study the properties of planetary objects outside of the solar system. To date, well over 100 such planets have been discovered and characterized, ${ }^{13}$ leading to much insight into the physical properties of planetary systems (e.g., see the recent review by Rauer 2011). In addition, over a thousand strong candidates from Kepler have been identified (Borucki et al. 2011), greatly expanding our understanding of several aspects of planetary systems, such as the properties of multi-planet systems (Latham et al. 2011; Lissauer et al. 2011), and the distribution of planetary radii (Howard et al. 2011). However, due to the large number of important variables that influence the physical properties of a planet (e.g., its mass, composition, age, irradiation, and tides, to name a few), we are still far from an empirically tested, comprehensive understanding of the formation and evolution of planetary systems.

\footnotetext{
* Based in part on observations obtained at the W. M. Keck Observatory, which is operated by the University of California and the California Institute of Technology. Keck time has been granted by NOAO (A289Hr) and NASA (N167Hr and N029Hr). Based in part on data collected at the Subaru Telescope, which is operated by the National Astronomical Observatory of Japan. Based in part on observations made with the Nordic Optical Telescope, operated on the island of La Palma jointly by Denmark, Finland, Iceland, Norway, and Sweden, in the Spanish Observatorio del Roque de los Muchachos of the Instituto de Astrofisica de Canarias.

12 Alfred. P. Sloan Research Fellow.

13 See, e.g., http://exoplanets.org (Wright et al. 2011) for the list of published planets, or www.exoplanet.eu (Schneider et al. 2011) for a more extended compilation, including unpublished results.
}

Here we present the discovery of four new TEPs identified by the Hungarian-made Automated Telescope Network (HATNet; Bakos et al. 2004) survey that contribute to the rapidly growing sample of TEPs. These planets transit relatively bright stars facilitating detailed characterization of their properties, such as measurements of their masses via radial velocity (RV) observations of the host star, or measuring their orbital tilt via the Rossiter-McLaughlin (RM) effect.

The HATNet survey for TEPs around bright stars $(9 \lesssim$ $r \lesssim 14.5)$ operates six wide-field instruments: four at the Fred Lawrence Whipple Observatory (FLWO) in Arizona (HAT-5, $-6,-7$, and -10 ), and two on the roof of the hangar servicing the Smithsonian Astrophysical Observatory's Submillimeter Array, in Hawaii (HAT-8 and -9). Since 2006, HATNet has announced and published 33 TEPs (e.g., Johnson et al. 2011). In this work we report our thirty-fourth through thirty-seventh discoveries, around the stars GSC 1622-01261, GSC 0203-01079, GSC 3020-02221, and GSC 3553-00723.

In Section 2 we summarize the detection of the photometric transit signals and the subsequent spectroscopic and photometric observations of each star to confirm the planets. In Section 3 we analyze the data to determine the stellar and planetary parameters. The properties of these planets are briefly discussed in Section 4.

\section{OBSERVATIONS}

The observational procedure employed by HATNet to discover TEPs has been described in detail in several previous discovery papers (e.g., Bakos et al. 2010; Latham et al. 2009). 
Table 1

Summary of Photometric Observations

\begin{tabular}{|c|c|c|c|c|}
\hline Instrument/Field & $\begin{array}{l}\text { Date } \\
(\mathrm{s})\end{array}$ & $\begin{array}{c}\text { Number of } \\
\text { Images }\end{array}$ & $\begin{array}{l}\text { Cadence } \\
\quad(\mathrm{sec})\end{array}$ & Filter \\
\hline \multicolumn{5}{|l|}{ HAT-P-34 } \\
\hline HAT-7/G293 & 2008 Oct-2009 May & 755 & 330 & $r$ \\
\hline HAT-8/G293 & 2008 Sep-2008 Dec & 2611 & 330 & $r$ \\
\hline HAT-6/G341 & 2007 Sep-2007 Dec & 1949 & 330 & $R$ \\
\hline HAT-9/G341 & 2007 Sep-2007 Nov & 2379 & 330 & $R$ \\
\hline KeplerCam & 2010 May 21 & 263 & 60 & $z$ \\
\hline KeplerCam & 2010 Oct 10 & 530 & 30 & $i$ \\
\hline \multicolumn{5}{|l|}{ HAT-P-35 } \\
\hline HAT-5/G364 & 2009 May & 21 & 330 & $r$ \\
\hline HAT-9/G364 & 2008 Dec-2009 May & 3155 & 330 & $r$ \\
\hline KeplerCam & 2011 Jan 16 & 110 & 100 & $i$ \\
\hline FTN & 2011 Jan 23 & 185 & 45 & $i$ \\
\hline KeplerCam & 2011 Mar 8 & 268 & 60 & $i$ \\
\hline \multicolumn{5}{|l|}{ HAT-P-36 } \\
\hline HAT-5/G143 & 2010 Apr-2010 Jul & 4471 & 210 & $r$ \\
\hline HAT-8/G143 & 2010 Apr-2010 Jul & 6262 & 210 & $r$ \\
\hline KeplerCam & 2010 Dec 24 & 131 & 130 & $i$ \\
\hline KeplerCam & 2011 Feb 3 & 101 & 100 & $i$ \\
\hline KeplerCam & 2011 Feb 7 & 105 & 100 & $i$ \\
\hline KeplerCam & 2011 Feb 15 & 186 & 60 & $i$ \\
\hline \multicolumn{5}{|l|}{ HAT-P-37 } \\
\hline HAT-7/G115 & 2009 Sep-2010 Jul & 7102 & 210 & $r$ \\
\hline HAT-9/G115 & 2008 Aug-2008 Sep & 2293 & 330 & $R$ \\
\hline KeplerCam & 2011 Feb 23 & 37 & 165 & $i$ \\
\hline KeplerCam & 2011 Mar 23 & 73 & 134 & $i$ \\
\hline KeplerCam & 2011 Apr 6 & 102 & 134 & $i$ \\
\hline
\end{tabular}

In the following subsections we highlight specific details of this procedure that are pertinent to the discoveries of the four planets presented in this paper.

\subsection{Photometric Detection}

Table 1 summarizes the HATNet discovery observations of each new planetary system. The HATNet images were processed and reduced to trend-filtered light curves following the procedure described by Bakos et al. (2010) and Pál (2009b). The light curves were searched for periodic box-shaped signals using the Box Least-Squares (BLS; see Kovács et al. 2002) method. We detected significant signals in the light curves of the stars summarized in Table 2; see also Figure 1.
Table 3

Summary of Reconnaissance Spectroscopy Observations ${ }^{\mathrm{a}}$

\begin{tabular}{cccc}
\hline \hline Instrument & HJD & $\begin{array}{c}\gamma_{\mathrm{RV}}^{\mathrm{b}} \\
\left(\mathrm{km} \mathrm{s}^{-1}\right)\end{array}$ & CC Peak \\
\hline HAT-P-34 & & & \\
TRES & 2454935.00839 & -47.81 & 0.726 \\
TRES & 2454966.97204 & -49.19 & 0.939 \\
TRES & 2454998.97956 & -49.12 & 0.819 \\
HAT-P-35 & & & \\
TRES & 2455289.64284 & 41.24 & 0.863 \\
TRES & 2455291.62482 & 40.54 & 0.883 \\
TRES & 2455320.64182 & 40.83 & 0.935 \\
TRES & 2455321.64383 & 41.08 & 0.940 \\
\hline
\end{tabular}

Notes.

a For HAT-P-36 and HAT-P-37, which were confirmed using the TRES spectrograph, there is no clear distinction between reconnaissance and highprecision observations. We do not list the results from the analysis of the TRES spectra for these targets here, these are instead described in Section 2.3.

b The heliocentric RV of the target in the IAU system, and corrected for the orbital motion of the planet.

c The peak value of the cross-correlation function between the observed spectrum and the best-matching synthetic template spectrum (normalized to be between 0 and 1). Observations with a peak height closer to 1.0 generally correspond to higher $\mathrm{S} / \mathrm{N}$ spectra.

\subsection{Reconnaissance Spectroscopy}

High-resolution, low-S/N "reconnaissance" spectra were obtained for HAT-P-34 and HAT-P-35 using the Tillinghast Reflector Echelle Spectrograph (TRES; Fúrész 2008) on the $1.5 \mathrm{~m}$ Tillinghast Reflector at FLWO. These observations were reduced and analyzed following the procedure described by Quinn et al. (2012) and Buchhave et al. (2010); the results are listed in Table 3. For both objects the spectra were single-lined, and showed RV variations on the order of $\sim 100 \mathrm{~m} \mathrm{~s}^{-1}$. Proper phasing of the RV with the photometric ephemeris gives confidence in acquiring further, high signal-to-noise spectroscopic observations to refine the orbit (see Section 2.3). While for HAT-P-34 the variations initially did not appear to phase with the photometric ephemeris, we entertained the possibility of a very significant non-zero eccentricity, and pursued follow-up of the target. For HAT-P-35 the variations were in phase with the photometric ephemeris indicating a $\sim 2.7 M_{\mathrm{J}}$ companion. For both HAT-P-36 and HAT-P-37 we obtained two TRES spectra near each of the predicted quadrature phases. For both objects the

Table 2

Summary of Discovery Data

\begin{tabular}{|c|c|c|c|c|c|c|c|}
\hline Host Planet & GSC & 2MASS & $\begin{array}{c}\text { R.A. } \\
\text { (HH:MM:SS) }\end{array}$ & $\begin{array}{c}\text { Decl. } \\
\text { (DD:MM:SS) }\end{array}$ & $\begin{array}{c}\mathrm{V}^{\mathrm{a}} \\
(\mathrm{mag})\end{array}$ & $\begin{array}{l}\text { Depth }^{\mathrm{b}} \\
\text { (mmag) }\end{array}$ & $\begin{array}{l}\text { Period } \\
\text { (days) }\end{array}$ \\
\hline HAT-P-34 & $1622-01261$ & $20124688+1806175$ & $20^{\mathrm{h}} 12^{\mathrm{m}} 46^{\mathrm{s}} .80$ & $+18^{\circ} 06^{\prime} 17^{\prime \prime} .5$ & $10.162 \pm 0.073$ & 7.9 & 5.4527 \\
\hline HAT-P-36 & $3020-02221$ & $12330390+4454552$ & $12^{\mathrm{h}} 33^{\mathrm{m}} 03^{\mathrm{s}} .96$ & $+44^{\circ} 54^{\prime} 55^{\prime \prime} .3$ & $12.262 \pm 0.068$ & 14.7 & 1.3273 \\
\hline HAT-P-37 & $3553-00723$ & $18571105+5116088$ & $18^{\mathrm{h}} 57^{\mathrm{m}} 11^{\mathrm{s}} .16$ & $+51^{\circ} 16^{\prime} 08^{\prime \prime} .9$ & $13.23 \pm 0.32^{\mathrm{c}}$ & 18.1 & 2.7974 \\
\hline
\end{tabular}

Notes.

${ }^{\text {a }}$ From Droege et al. 2006.

b Note that the apparent depth of the HATNet transit for all four targets is shallower than the true transit depth due to blending with unresolved neighbors in the low spatial resolution HATNet images (the median FWHM of the point-spread function at the center of a HATNet image is $\sim 25^{\prime \prime}$ ). Also, we applied the trend-filtering procedure in non-signal-reconstructive mode, which reduces the transit depth while increasing the signal-to-noise ratio of the detection. For each system, the ratio of the planet and stellar radii, which is related to the true transit depth, is determined in Section 3.2 using the higher spatial resolution photometric follow-up observations described in Section 2.4.

${ }^{\mathrm{c}}$ From Lasker et al. 2008. 

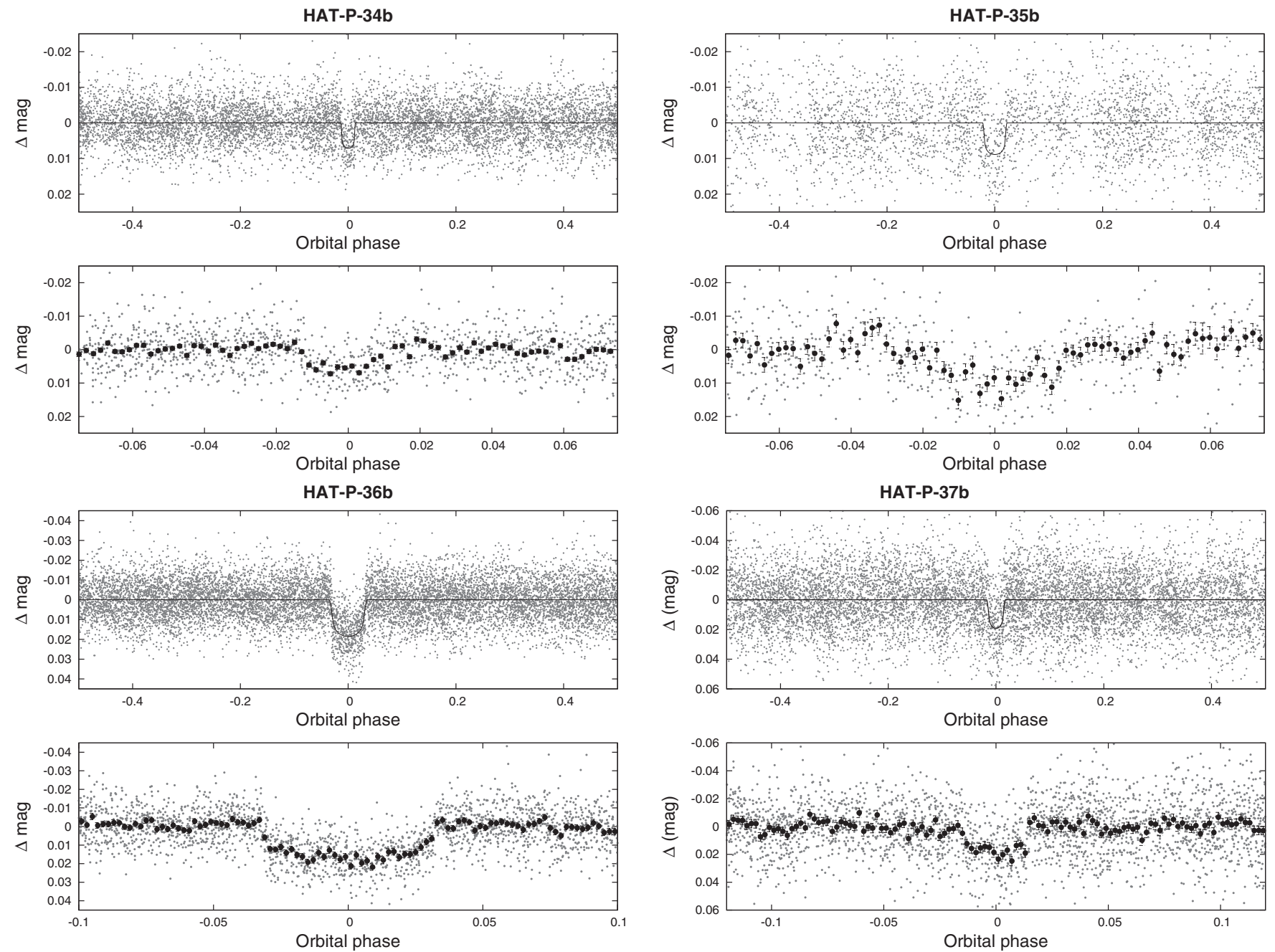

Figure 1. HATNet light curves of HAT-P-34 through HAT-P-37. See Table 2 for a summary of the observations. For each planet we show two panels. The top panel shows the unbinned light curve folded with the period resulting from the global fit described in Section 3. The solid line shows the model fit to the light curve (Section 3.2). The bottom panel shows the region zoomed-in on the transit. The dark filled circles show the light curve binned in phase with a bin size of 0.002 .

spectra were single-lined. For HAT-P-36 the resulting RV measurements showed $\sim 400 \mathrm{~m} \mathrm{~s}^{-1}$ variation in phase with the photometric ephemeris, while for HAT-P-37 the RV measurements showed $\sim 260 \mathrm{~m} \mathrm{~s}^{-1}$ variation in phase with the ephemeris. We opted to continue observing both of these objects using TRES with the aim of confirming the planets. The TRES observations of HAT-P-36 and HAT-P-37 are discussed further in the following subsection.

\subsection{High Resolution, High-S/N Spectroscopy}

We proceeded with the follow-up of each candidate by obtaining high-resolution, high-S/N spectra to characterize the RV variations, and to refine the determination of the stellar parameters. These observations are summarized in Table 4. The RV measurements and uncertainties for HAT-P-34 through HAT-P-37 are given in the Appendix. The period-folded data, along with our best fit described below in Section 3, are displayed in Figures 2-5.

Four facilities were used in the confirmation of these planets (including three separate facilities used for HAT-P-34). These facilities are HIRES (Vogt et al. 1994) on the $10 \mathrm{~m}$ Keck I telescope in Hawaii, the High-Dispersion Spectrograph (HDS; Noguchi et al. 2002) on the $8.3 \mathrm{~m}$ Subaru telescope in Hawaii,
Table 4

Summary of High-SN Spectroscopic Observations Used in Measuring the Orbits

\begin{tabular}{lcc}
\hline \hline Instrument & Date(s) & $\begin{array}{c}\text { Number of } \\
\text { RV Obs. }\end{array}$ \\
\hline HAT-P-34 & 2010 May & 6 \\
$\quad$ Subaru/HDS & 2010 Jun-2010 Sep & 14 \\
Keck/HIRES & 2010 Jul-2010 Aug & 10 \\
$\quad$ NOT/FIES & & \\
HAT-P-35 & 2010 Sep-2010 Dec & 7 \\
$\quad$ Keck/HIRES & 2010 Oct & $5^{\text {a }}$ \\
$\quad$ NOT/FIES & & 12 \\
HAT-P-36 & 2010 Dec-2011 Jan & 13 \\
FLWO 1.5/TRES & & \\
HAT-P-37 & & \\
FLWO 1.5/TRES & 2011 Mar-2011 May & 13 \\
\hline
\end{tabular}

Notes. ${ }^{a}$ One of the NOT/FIES spectra of HAT-P-35 was aborted early due to morning twilight and high humidity, another exposure was obtained partly during transit and may be affected by the Rossiter-McLaughlin effect. The remaining three NOT/FIES spectra do not provide sufficient phase coverage to constrain the orbit. We therefore do not include the velocities measured from these spectra in the analysis of HAT-P-35. 

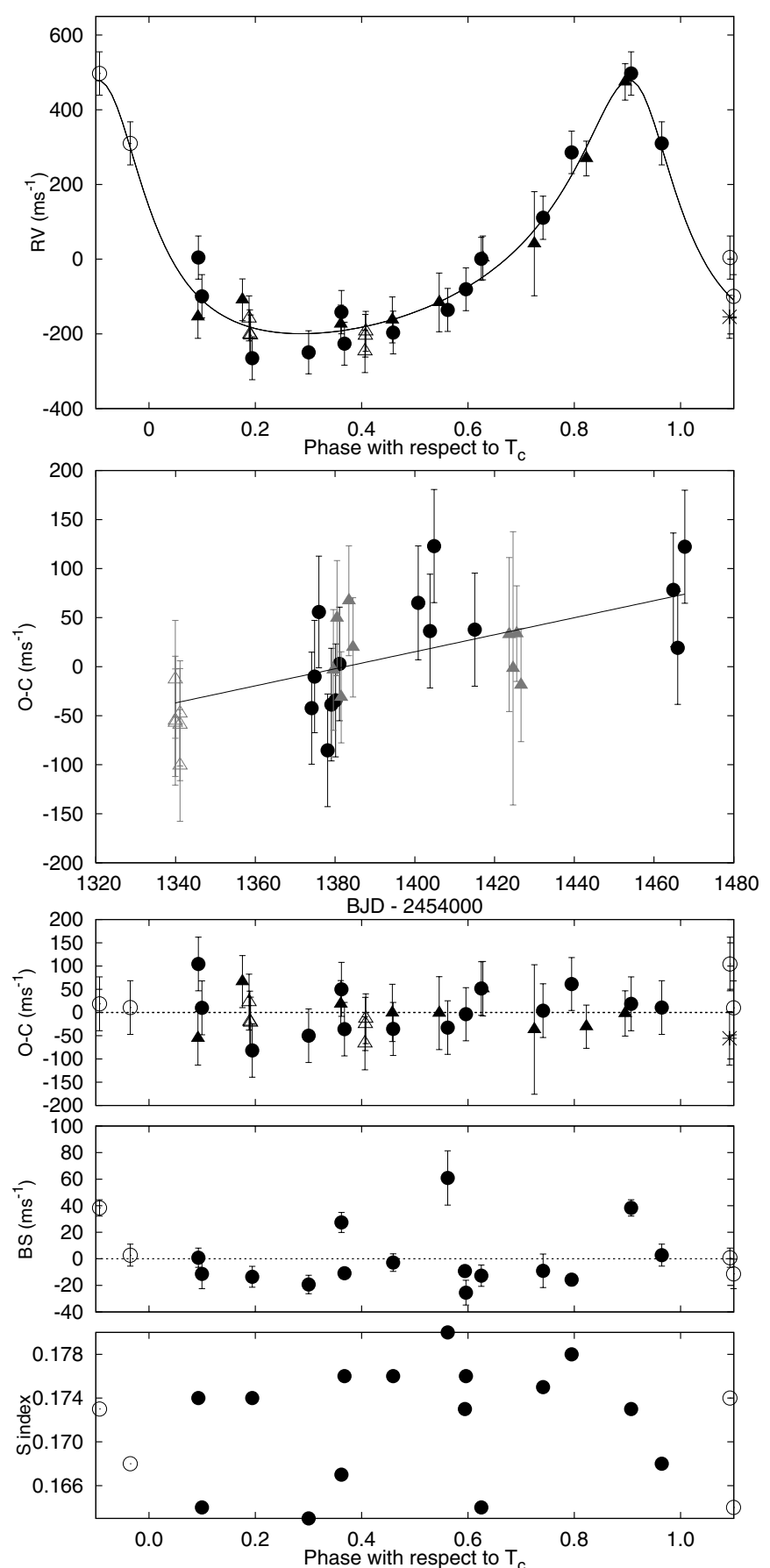

Figure 2. Top panel: high-precision RV measurements for HAT-P-34 shown as a function of orbital phase, along with our best-fit model (see Table 6). Open triangles show measurements from Subaru/HDS, filled circles show measurements from Keck/HIRES, and filled triangles show measurements from NOT/FIES. Zero phase corresponds to the time of mid-transit. The center-ofmass velocity and a linear trend have been subtracted. Second panel: velocity $O-C$ residuals from the best-fit single Keplerian orbit model as a function of time. The residuals show a slight linear trend, possibly indicating a third body in the system. Note that the zero points of the three separate instruments are independently free parameters. Third panel: velocity $O-C$ residuals from the best fit including both the Keplerian orbit and linear trend, shown as a function of orbital phase. The error bars include a jitter term $\left(56.0 \mathrm{~m} \mathrm{~s}^{-1}\right.$ for the Keck/ HIRES observations, and $32.0 \mathrm{~m} \mathrm{~s}^{-1}$ for the Subaru/HDS observations; no jitter has been added to the NOT/FIES RV uncertainties) added in quadrature to the formal errors (see Section 3.2). Fourth panel: bisector spans (BS) from Keck/HIRES, with the mean value subtracted. The measurement from the template spectrum is included. Bottom panel: chromospheric activity index $S$ measured from the Keck spectra. Note the different vertical scales of the panels. Observations shown twice are represented with open symbols.

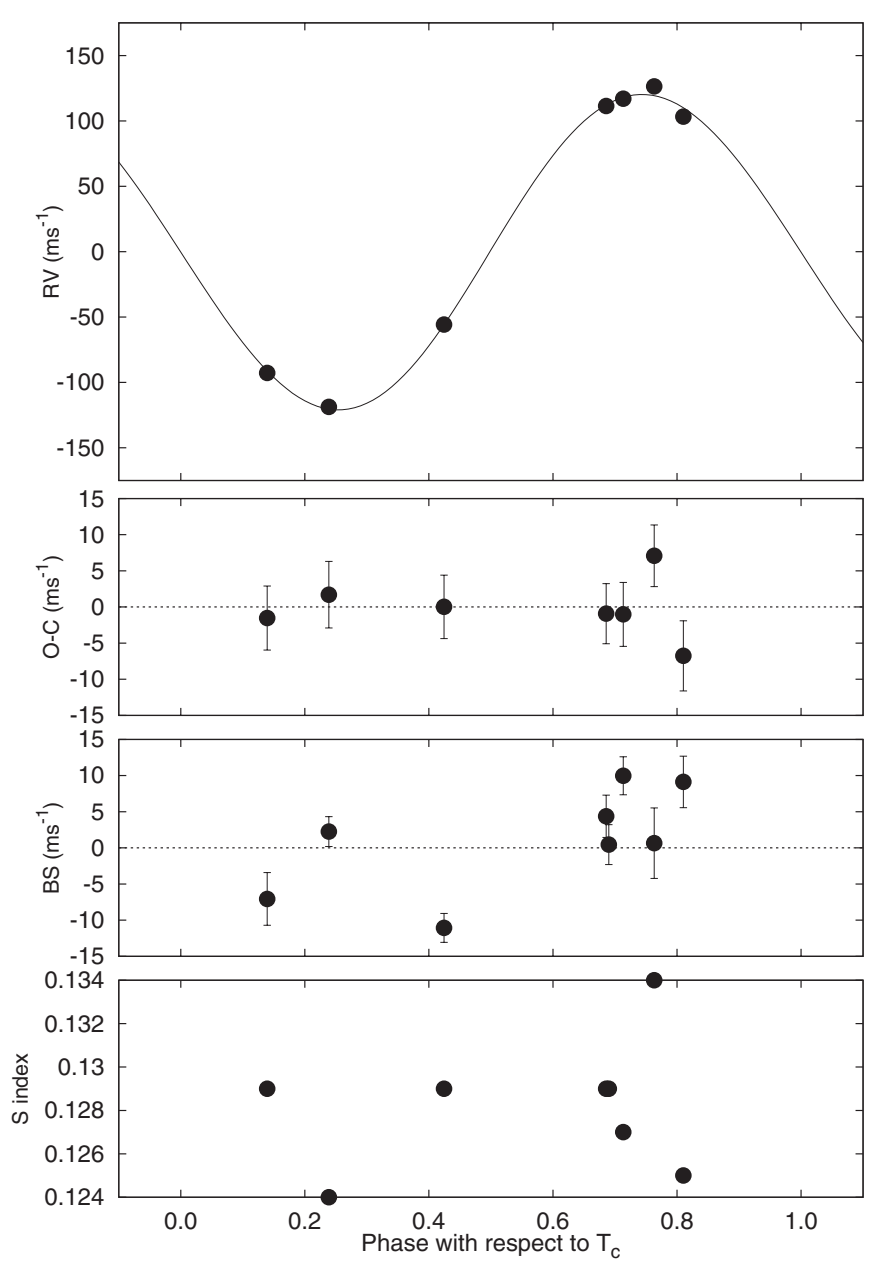

Figure 3. Keck/HIRES observations of HAT-P-35. The panels are as in Figure 2. The parameters used in the best-fit model are given in Table 6.

the FIbre-fed Échelle Spectrograph (FIES) on the $2.5 \mathrm{~m}$ Nordic Optical Telescope (NOT) at La Palma, Spain (Djupvik \& Andersen 2010), and TRES on the FLWO $1.5 \mathrm{~m}$ telescope.

The HIRES and HDS observations made use of the iodine-cell method (Marcy \& Butler 1992; Butler et al. 1996) for precise wavelength calibration and relative $\mathrm{RV}$ determination, while the FIES and TRES observations made use of Th-Ar lamp spectra obtained before and after the science exposures. The HIRES observations were reduced to relative RVs in the barycentric frame following Butler et al. (1996), Johnson et al. (2009), and Howard et al. (2010); the HDS observations were reduced following Sato et al. (2002, 2005); and the FIES and TRES observations were reduced following Buchhave et al. (2010).

We found that for all four systems the RV residuals from the best-fit models, described below in Section 3.2, exhibit excess scatter over what is expected based on the formal measurement uncertainties. Such excess scatter, or "jitter" has been well known for stars, and can stem from multiple sources. The excess is in the residuals of the observations with respect to a physical (and possibly instrumental) model. If this model is not adequate, the residuals can be larger than expected. For example, in the case of HAT-P-34b, ignoring the linear trend in the RVs would lead to a much increased "jitter." Additional planets may cause jitter, as the limited number of RV observations is not enough to uniquely identify and model such systems. The typical source of the jitter, however, is the star itself, namely inhomogeneities 


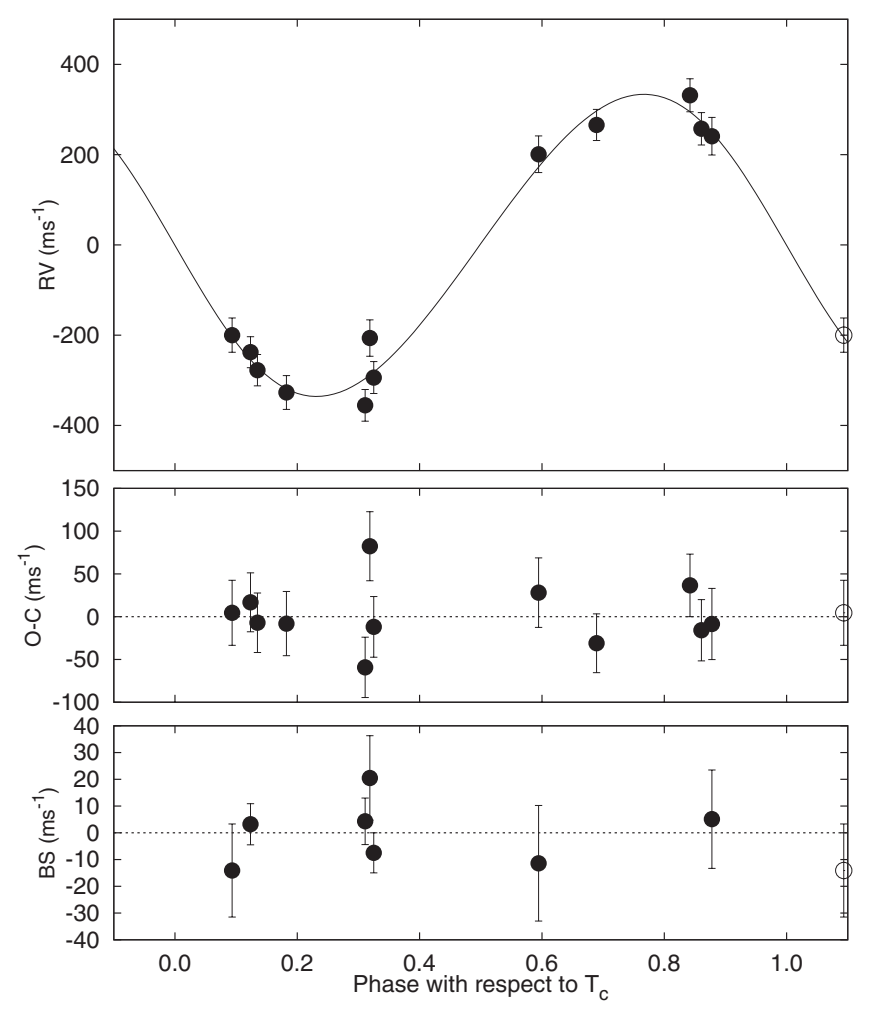

Figure 4. FLWO $1.5 \mathrm{~m}$ /TRES observations of HAT-P-36. The panels are as in Figure 2. The $S$ index is not available for these observations. The parameters used in the best-fit model are given in Table 6.

(spots, flares, plages, etc.) on the stellar surface (e.g., Makarov et al. 2009; Martínez-Arnáiz et al. 2010) causing jitters up to $100 \mathrm{~ms}$. Granulation and stellar oscillations contribute on a smaller scale, but are present for non-active stars that are outside the instability strip. A recent publication by Cegla et al. (2012) discusses the stellar jitter due to variable gravitational redshift of the star, as the stellar radius changes due to oscillations $(\Delta R$ of $10^{-4}$ causing $\left.\sim 0.1 \mathrm{~m} \mathrm{~s}^{-1}\right)$. And, of course, systematics in the instrument further inflate the jitter. A review of RV jitter of stars observed by the Keck telescope is given in Wright (2005).

In order to ensure realistic estimates of the system parameter uncertainties we add in quadrature an RV jitter to the formal RV measurement uncertainties such that $\chi^{2}$ per degree of freedom is unity for the best-fit model for each planet. We adopt an independent jitter for the observations made by each instrument of each planet. The RV uncertainties given in the tables presented in the Appendix do not include this jitter; we do include the jitter in Figures 2-5.

For HAT-P-34 and HAT-P-35 we also show the $S$ index, which is a measure of the chromospheric activity of the star derived from the flux in the cores of the $\mathrm{Ca} I \mathrm{H}$ and $\mathrm{K}$ lines. This index was computed following Isaacson \& Fischer (2010) and has been calibrated to the scale of Vaughan et al. (1978). A procedure for obtaining calibrated $S$ index values from the TRES spectra has not yet been developed, so we do not provide these measurements for HAT-P-36 or HAT-P-37. We convert the $S$ index values to $\log R_{\mathrm{HK}}^{\prime}$ following Noyes et al. (1984) and find median values of $\log R_{\mathrm{HK}}^{\prime}=-4.859$ and $\log R_{\mathrm{HK}}^{\prime}=-5.242$ for HAT-P-34 and HAT-P-35, respectively. These values imply that neither star has a particularly high level of chromospheric activity.

Following Queloz et al. (2001) and Torres et al. (2007), we checked whether the measured radial velocities are not real, but

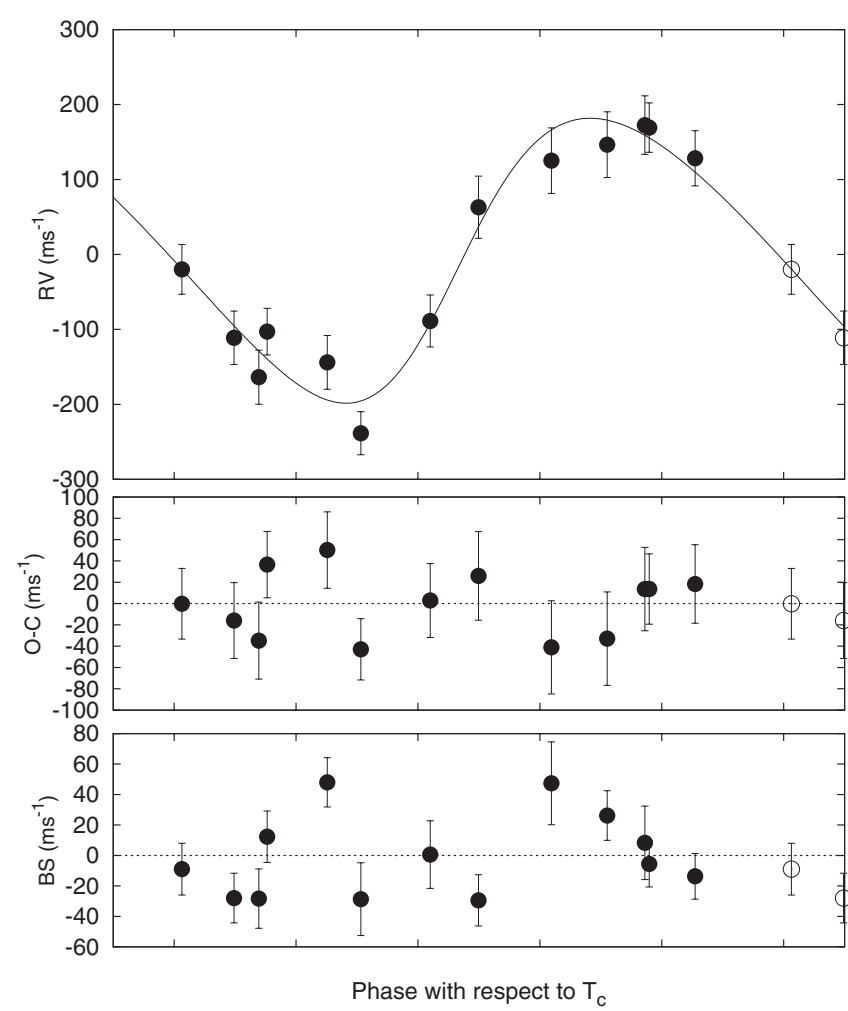

Figure 5. FLWO $1.5 \mathrm{~m}$ /TRES observations of HAT-P-37. The panels are as in Figure 2. The $S$ index is not available for these observations. The parameters used in the best-fit model are given in Table 6.

are instead caused by distortions in the spectral line profiles due to contamination from a nearby unresolved eclipsing binary. A bisector (BS) analysis for each system based on the Keck and TRES spectra was done as described in Section 5 of Bakos et al. (2007a). For HAT-P-35, which is relatively faint, we found that the measured BSs were significantly affected by scattered moonlight and applied an empirical correction for this effect following Hartman et al. (2009; see also Kovács et al. 2010). For HAT-P-34 the BS scatter is fairly high $\left(\sim 25 \mathrm{~m} \mathrm{~s}^{-1}\right)$, but this is in line with the high $\mathrm{RV}$ jitter $\left(\sim 60 \mathrm{~m} \mathrm{~s}^{-1}\right)$, which is typical of an F star with $v \sin i=24.0 \pm 0.5 \mathrm{~km} \mathrm{~s}^{-1}$ (Saar et al. 2003; Hartman et al. 2011b).

None of the systems show significant bisector span variations (relative to the semi-amplitude of the RV variations) that phase with the photometric ephemeris. Such variations are generally expected if the transit and RV signals were due to blends rather than planets. While the lack of bisector span variations does not exclude all blend scenarios, it does significantly limit the possible blend scenarios that can reproduce our current data within the measurement errors, i.e., configurations that are compatible with the photometric and spectroscopic observations, proper motions, color indices, and moderately high resolution imaging. We have found in the past that invoking detailed blend modeling to exclude all possible blend configurations and confirm the planet hypothesis (e.g., Hartman et al. 2011a, Sections 3.2.2 and 3.2.3) is rarely of any incremental value when the ingress and egress durations are short relative to the total transit duration, the RV variations exhibit a Keplerian orbit in phase with the photometric ephemeris, and bisector spans show no correlation with the orbit. We conclude that the velocity variations detected for all four stars are real, and that each star is orbited by a close-in giant planet. 


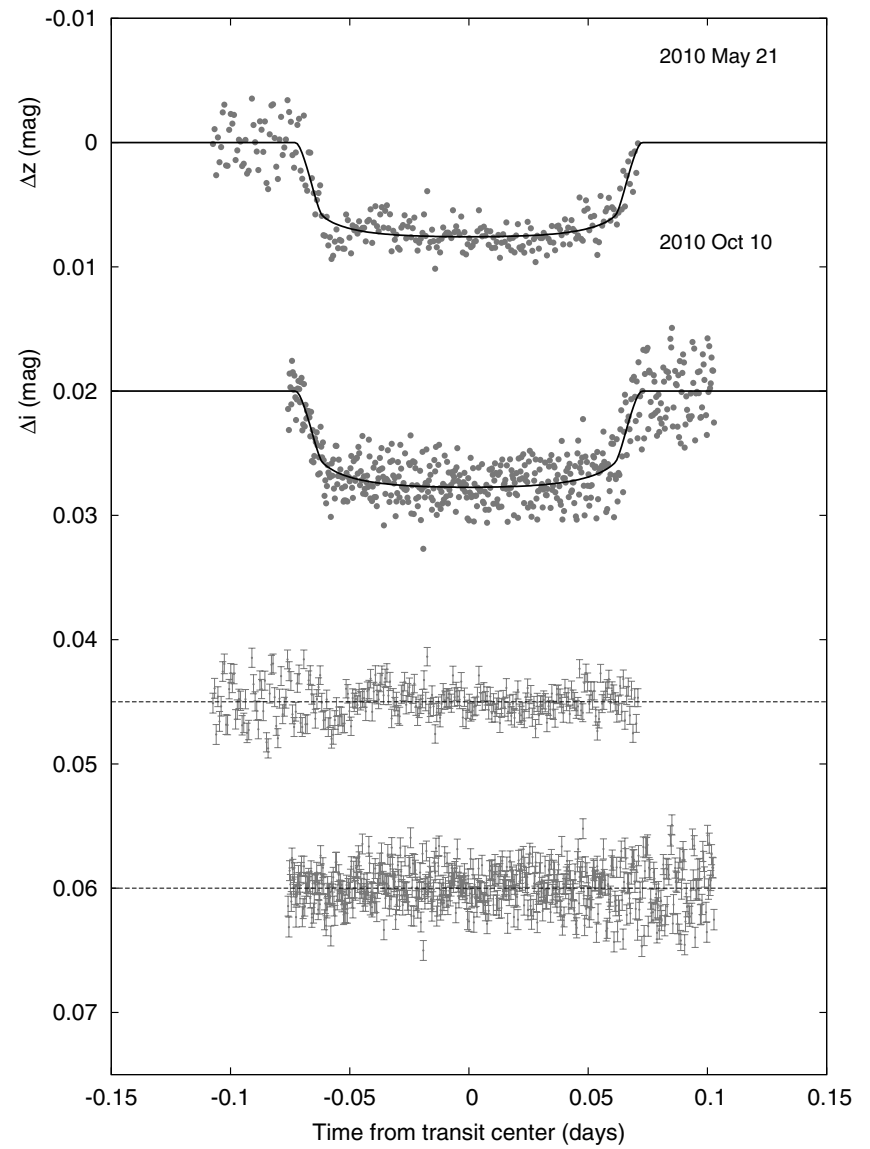

Figure 6. Unbinned transit light curves for HAT-P-34, acquired with KeplerCam at the FLWO $1.2 \mathrm{~m}$ telescope. The light curves have been EPD- and TFAprocessed, as described in Section 3.2. The dates of the events are indicated. Curves after the first are displaced vertically for clarity. Our best fit from the global modeling described in Section 3.2 is shown by the solid lines. Residuals from the fits are displayed at the bottom, in the same order as the top curves. The error bars represent the photon and background shot noise, plus the readout noise.

\subsection{Photometric Follow-up Observations}

In order to permit a more accurate modeling of the light curves, we conducted additional photometric observations using the KeplerCam CCD camera on the FLWO $1.2 \mathrm{~m}$ telescope, and the Spectral Instrument CCD on the $2.0 \mathrm{~m}$ Faulkes Telescope North (FTN) at Haleakala Observatory in Hawaii, which is operated by the Las Cumbres Observatory Global Telescope (LCOGT). The observations for each target are summarized in Table 1.

The reduction of these images was performed as described by Bakos et al. (2010). We applied External Parameter Decorrelation (EPD; Bakos et al. 2010) and the Trend Filtering Algorithm (TFA; Kovács et al. 2005) to remove trends simultaneously with light-curve modeling. The final time series, together with our best-fit transit light-curve models, are shown in the top portion of Figures 6-9 for HAT-P-34 through HAT-P-37, respectively. The individual measurements, permitting independent analysis by other researchers, are reported in the Appendix.

\section{ANALYSIS}

\subsection{Properties of the Parent Star}

Stellar atmospheric parameters for HAT-P-34 and HAT-P-35 were measured using our template spectra obtained with the Keck/HIRES instrument, and the analysis package known as

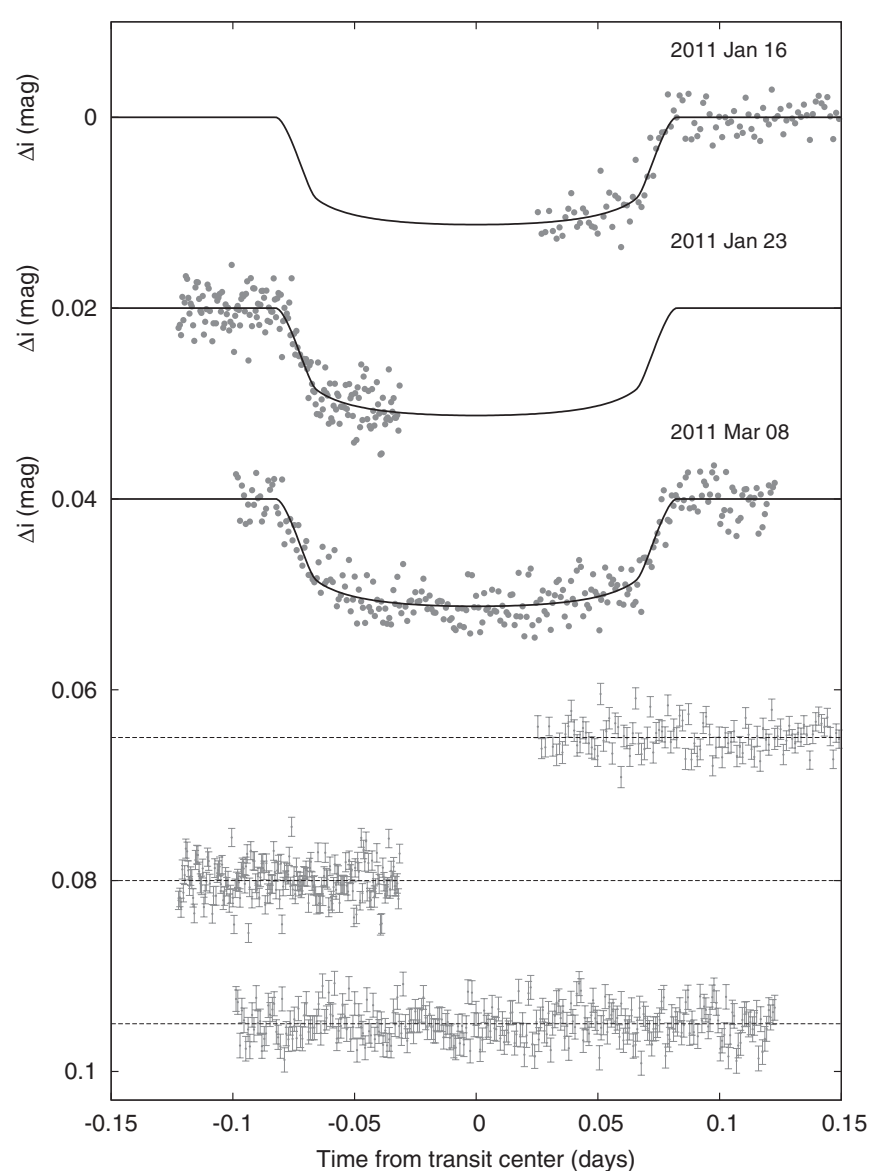

Figure 7. Similar to Figure 6; here we show the follow-up light curves for HAT-P-35.

Spectroscopy Made Easy (SME; Valenti \& Piskunov 1996), along with the atomic line database of Valenti \& Fischer (2005). For HAT-P-36 and HAT-P-37 the stellar atmospheric parameters were determined by cross-correlating the TRES observations against a finely sampled grid of synthetic spectra based on Kurucz (2005) model atmospheres. This procedure, known as Stellar Parameter Classification (SPC), will be described in detail in a forthcoming paper (L. A. Buchhave et al., in preparation). We note that SPC has been performed in the past on numerous HATNet transiting planet candidates (Buchhave, personal communication), and the results were consistent with those of SME.

For each star, we obtained the following initial spectroscopic parameters and uncertainties:

1. HAT-P-34-effective temperature $T_{\text {eff } \star}=6400 \pm 100 \mathrm{~K}$, metallicity $[\mathrm{Fe} / \mathrm{H}]=+0.21 \pm 0.1 \mathrm{dex}$, stellar surface gravity $\log g_{\star}=3.98 \pm 0.1(\mathrm{cgs})$, and projected rotational velocity $v \sin i=24.5 \pm 1.0 \mathrm{~km} \mathrm{~s}^{-1}$.

2. HAT-P-35-effective temperature $T_{\mathrm{eff} \star}=5940 \pm 88 \mathrm{~K}$, metallicity $[\mathrm{Fe} / \mathrm{H}]=+0.01 \pm 0.08 \mathrm{dex}$, stellar surface gravity $\log g_{\star}=3.98 \pm 0.1$ (cgs), and projected rotational velocity $v \sin i=0.5 \pm 0.5 \mathrm{~km} \mathrm{~s}^{-1}$.

3. HAT-P-36-effective temperature $T_{\mathrm{eff} \star}=5850 \pm 100 \mathrm{~K}$, metallicity $[\mathrm{Fe} / \mathrm{H}]=+0.38 \pm 0.1 \mathrm{dex}$, stellar surface gravity $\log g_{\star}=4.73 \pm 0.17$ (cgs), and projected rotational velocity $v \sin i=2.86 \pm 0.5 \mathrm{~km} \mathrm{~s}^{-1}$.

4. HAT-P-37-effective temperature $T_{\mathrm{eff} \star}=5570 \pm 100 \mathrm{~K}$, metallicity $[\mathrm{Fe} / \mathrm{H}]=+0.09 \pm 0.1 \mathrm{dex}$, stellar surface gravity $\log g_{\star}=4.67 \pm 0.1(\mathrm{cgs})$, and projected rotational velocity $v \sin i=2.95 \pm 0.5 \mathrm{~km} \mathrm{~s}^{-1}$. 


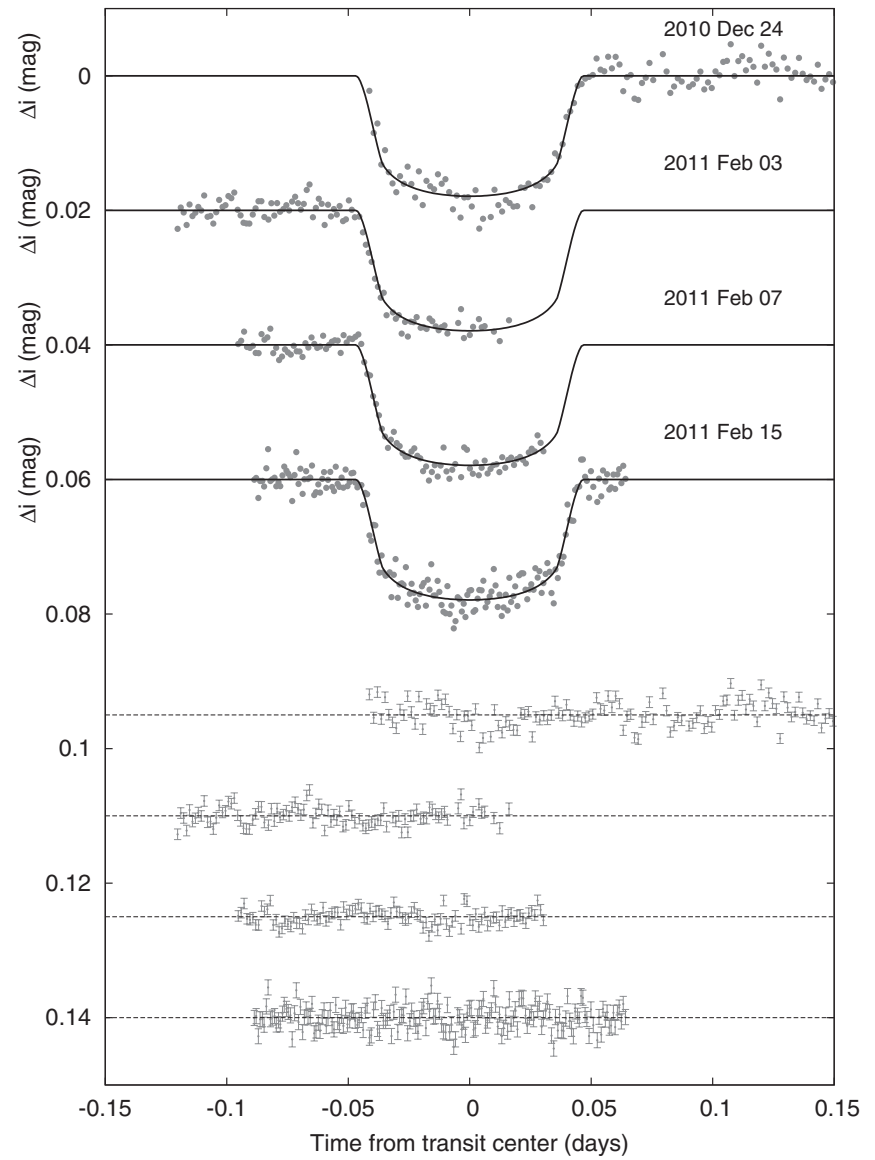

Figure 8. Similar to Figure 6; here we show the follow-up light curves for HAT-P-36.

Following Bakos et al. (2010), these initial values of $T_{\mathrm{eff} \star}$, $\log g_{\star}$, and $[\mathrm{Fe} / \mathrm{H}]$ were used to determine the quadratic limbdarkening coefficients needed in the global modeling of the follow-up photometry (summarized in Section 3.2). This analysis yields $\rho_{\star}$, the mean stellar density, which is closely related to $a / R_{\star}$, the normalized semimajor axis, and provides a tighter constraint on the stellar parameters than does the spectroscopically determined $\log g_{\star}$ (e.g., Sozzetti et al. 2007). We combined $\rho_{\star}, T_{\text {eff } \star}$, and $[\mathrm{Fe} / \mathrm{H}]$ with stellar evolution models from the Yonsei-Yale (YY) series by Yi et al. (2001) to determine probability distributions of other stellar properties, including $\log g_{\star}$. For each system we carried out a second SME or SPC iteration in which we adopted the new value of $\log g_{\star}$ so determined and held it fixed in a new SME or SPC analysis, adjusting only $T_{\text {eff }}$, $[\mathrm{Fe} / \mathrm{H}]$, and $v \sin i$, followed by a second global modeling of the RV and light curves, together with improved limb darkening parameters. The final atmospheric parameters that we adopt, together with stellar parameters inferred from the YY models (such as the mass, radius and age) are listed in Table 5 for all four stars.

The inferred location of each star in a diagram of $a / R_{\star}$ versus $T_{\text {eff } \star}$, analogous to the classical H-R diagram, is shown in Figure 10. In each case the stellar properties and their $1 \sigma$ and $2 \sigma$ confidence ellipses are displayed against the backdrop of model isochrones for a range of ages, and the appropriate stellar metallicity. For comparison, the locations implied by the initial SME and SPC results are also shown (in each case with a triangle).

The stellar evolution modeling provides color indices that we compared against the measured values as a sanity check. For each star we used the near-infrared magnitudes from the

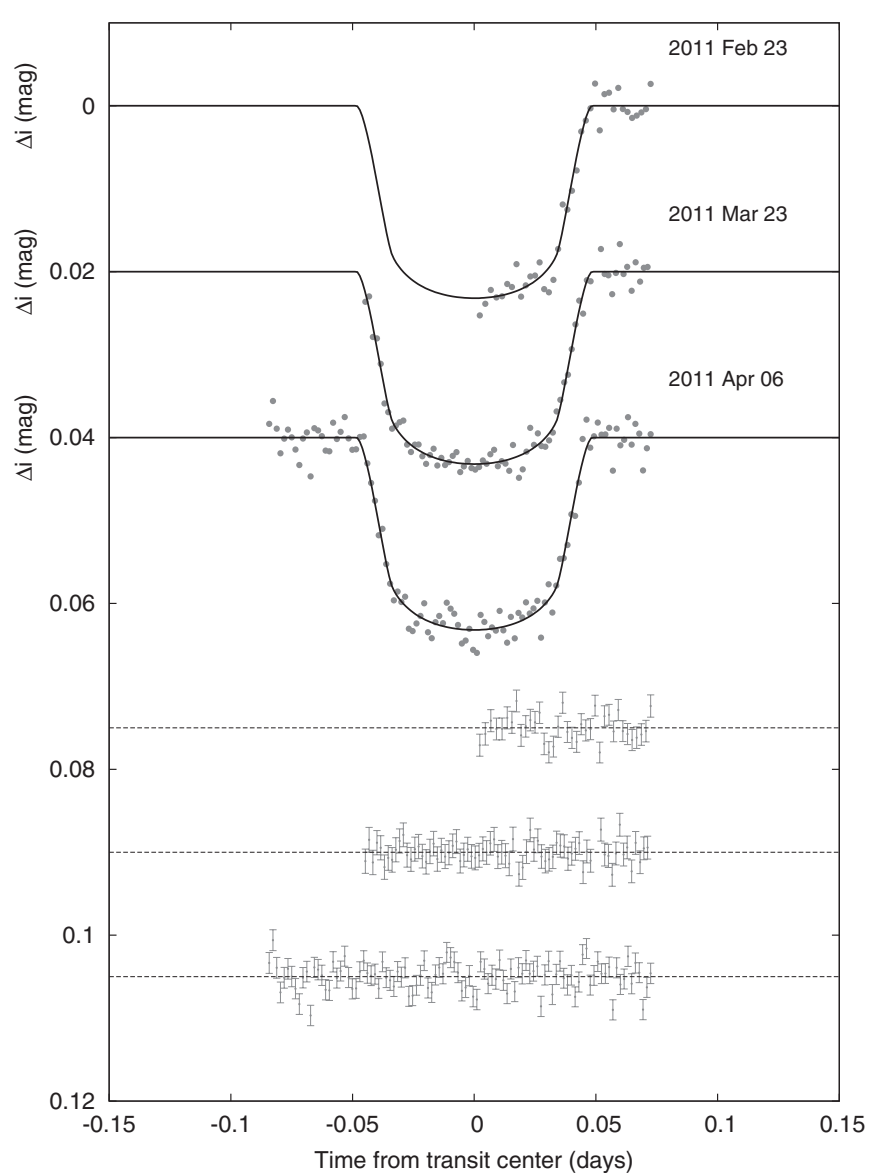

Figure 9. Similar to Figure 6; here we show the follow-up light curves for HAT-P-37.

Two Micron All Sky Survey (2MASS) Catalogue (Skrutskie et al. 2006), which are given in Table 5. These were converted to the photometric system of the models (ESO) using the transformations by Carpenter (2001). The resulting 2MASSbased color indices were all consistent (within $1 \sigma$ ) with the stellar model based color indices.

The distance for each star given in Table 5 was computed from the absolute $K$ magnitude from the models and the 2MASS $K_{S}$ magnitudes, ignoring extinction.

\subsection{Global Modeling of the Data}

We simultaneously modeled the HATNet photometry, the follow-up photometry, and the high-precision RV measurements using the procedures described by Bakos et al. (2010). Namely, the best fit was determined by a downhill simplex minimization, and was followed by a Monte Carlo Markov Chain run to scan the parameter space around the minimum, and establish the errors (Pál 2009b). For each system we used a Mandel \& Agol (2002) transit model, together with the EPD and TFA trend-filters, to describe the follow-up light curves, a Mandel \& Agol (2002) transit model for the HATNet light curve(s), and a Keplerian orbit using the formalism of Pál (2009a) for the RV curve(s). For HAT-P-34 we included a linear trend in the $\mathrm{RV}$ model, but find that it is only significant at the $\sim 2 \sigma$ level; the planet and stellar parameters are changed by less than $1 \sigma$ when the trend is not included in the fit. The parameters that we adopt for each system are listed in Table 6. In all cases we allow the eccentricity to vary so that the uncertainty on this parameter is propagated into the uncertainties on the other physical parameters, such as the stellar and planetary masses and 
Table 5

Stellar Parameters for HAT-P-34 through HAT-P-37

\begin{tabular}{|c|c|c|c|c|c|}
\hline Parameter & $\begin{array}{c}\text { HAT-P-34 } \\
\text { Value }\end{array}$ & $\begin{array}{l}\text { HAT-P-35 } \\
\text { Value }\end{array}$ & $\begin{array}{l}\text { HAT-P-36 } \\
\text { Value }\end{array}$ & $\begin{array}{c}\text { HAT-P-37 } \\
\text { Value }\end{array}$ & Source \\
\hline \multicolumn{6}{|l|}{ Spectroscopic properties } \\
\hline$T_{\text {eff } \star}(\mathrm{K}) \ldots$ & $6442 \pm 88$ & $6096 \pm 88$ & $5560 \pm 100$ & $5500 \pm 100$ & Spec. Analysis ${ }^{a}$ \\
\hline$[\mathrm{Fe} / \mathrm{H}] \ldots$ & $+0.22 \pm 0.04$ & $+0.11 \pm 0.08$ & $+0.26 \pm 0.10$ & $+0.03 \pm 0.10$ & Spec. Analysis \\
\hline$v \sin i\left(\mathrm{~km} \mathrm{~s}^{-1}\right) \ldots$ & $24.0 \pm 0.5$ & $0.5 \pm 0.5$ & $3.58 \pm 0.5$ & $3.07 \pm 0.5$ & Spec. Analysis \\
\hline$v_{\mathrm{mac}}\left(\mathrm{km} \mathrm{s}^{-1}\right) \ldots$ & 5.05 & 4.52 & 0.00 & $\ldots$ & Spec. Analysis \\
\hline$\gamma_{\mathrm{RV}}\left(\mathrm{km} \mathrm{s}^{-1}\right) \ldots$ & $-49.26 \pm 0.30$ & $40.95 \pm 0.20$ & $-16.29 \pm 0.10$ & $-20.53 \pm 0.1$ & TRES \\
\hline \multicolumn{6}{|l|}{ Photometric properties } \\
\hline$V(\operatorname{mag}) \ldots$ & $10.162 \pm 0.073$ & $12.46 \pm 0.11$ & $12.262 \pm 0.068$ & $13.23 \pm 0.32$ & TASS, GSC ${ }^{\mathrm{b}}$ \\
\hline$V-I_{C}(\mathrm{mag}) \ldots$ & $0.557 \pm 0.12$ & $0.662 \pm 0.12$ & $0.760 \pm 0.13$ & $\ldots$ & TASS \\
\hline$J$ (mag). . . & $9.460 \pm 0.022$ & $11.358 \pm 0.024$ & $11.046 \pm 0.027$ & $12.092 \pm 0.027$ & 2MASS \\
\hline$H(\mathrm{mag}) .$. & $9.322 \pm 0.030$ & $11.072 \pm 0.023$ & $10.723 \pm 0.030$ & $11.714 \pm 0.032$ & 2MASS \\
\hline$K_{s}(\mathrm{mag}) \ldots$ & $9.247 \pm 0.023$ & $11.030 \pm 0.021$ & $10.603 \pm 0.021$ & $11.667 \pm 0.020$ & 2MASS \\
\hline$M_{\star}\left(M_{\odot}\right) \ldots$ & $1.392 \pm 0.047$ & $1.236 \pm 0.048$ & $1.022 \pm 0.049$ & $0.929 \pm 0.043$ & $\mathrm{YY}+a / R_{\star}+$ Spec. Analysis ${ }^{\mathrm{c}}$ \\
\hline$R_{\star}\left(R_{\odot}\right) \ldots$ & $1.535_{-0.102}^{+0.135}$ & $1.435 \pm 0.084$ & $1.096 \pm 0.056$ & $0.877_{-0.044}^{+0.059}$ & $\mathrm{YY}+a / R_{\star}+\mathrm{Spec}$. Analysis \\
\hline $\log g_{\star}(\operatorname{cgs}) \ldots$ & $4.21 \pm 0.06$ & $4.21 \pm 0.04$ & $4.37 \pm 0.04$ & $4.52 \pm 0.05$ & $\mathrm{YY}+a / R_{\star}+$ Spec. Analysis \\
\hline$L_{\star}\left(L_{\odot}\right) \ldots$ & $3.63_{-0.51}^{+0.75}$ & $2.55_{-0.30}^{+0.40}$ & $1.03 \pm 0.15$ & $0.62_{-0.09}^{+0.11}$ & $\mathrm{YY}+a / R_{\star}+$ Spec. Analysis \\
\hline$M_{V}(\operatorname{mag}) \ldots$ & $3.32 \pm 0.19$ & $3.77 \pm 0.15$ & $4.83 \pm 0.17$ & $5.41 \pm 0.19$ & $\mathrm{YY}+a / R_{\star}+$ Spec. Analysis \\
\hline$M_{K}(\mathrm{mag} ; \mathrm{ESO}) \ldots$ & $2.24 \pm 0.17$ & $2.43 \pm 0.13$ & $3.14 \pm 0.12$ & $3.64 \pm 0.14$ & $\mathrm{YY}+a / R_{\star}+$ Spec. Analysis \\
\hline Age (Gyr)... & $1.7_{-0.5}^{+0.4}$ & $3.5_{-0.5}^{+0.8}$ & $6.6_{-1.8}^{+2.9}$ & $3.6_{-2.2}^{+4.1}$ & $\mathrm{YY}+a / R_{\star}+\mathrm{Spec}$. Analysis \\
\hline Distance $(\mathrm{pc}) \ldots$ & $257_{-17}^{+22}$ & $535 \pm 32$ & $317 \pm 17$ & $411 \pm 26$ & $\mathrm{YY}+a / R_{\star}+$ Spec. Analysis \\
\hline
\end{tabular}

Notes.

${ }^{a}$ Based on the analysis of high-resolution spectra. For HAT-P-34 and HAT-P-35 this corresponds to SME applied to iodine-free Keck/HIRES spectra, while for HAT-P-36 and HAT-P-37 this corresponds to SPC applied to the TRES spectra (Section 3.1). These parameters also have a small dependence on the iterative analysis incorporating the isochrone search and global modeling of the data, as described in the text.

${ }^{\mathrm{b}}$ For HAT-P-34 through HAT-P-36 the value is taken from the TASS catalog, while for HAT-P-37 the value is taken from the GSC version 2.3.2.

${ }^{\mathrm{c}} \mathrm{YY}+a / R_{\star}+$ Spec. Analysis $=$ Based on the YY isochrones (Yi et al. 2001), $a / R_{\star}$ as a luminosity indicator, and the spectroscopic analysis results.

radii; the observations of HAT-P-35b, HAT-P-36b, and HAT-P$37 \mathrm{~b}$ are consistent with these planets being on circular orbits.

\section{DISCUSSION}

We have presented the discovery of four new transiting planets. Below we briefly discuss their properties.

\subsection{HAT-P-34b}

HAT-P-34b is a relatively massive $M_{p}=3.328 \pm 0.211 M_{\mathrm{J}}$ planet on a relatively long-period $(P=5.452654 \pm$ 0.000016 days), eccentric ( $e=0.441 \pm 0.032)$ orbit. There are only five known transiting planets with higher eccentricities (HAT-P-2b, $e=0.5171 \pm 0.0033$, Pál et al. 2010; Bakos et al. 2007a; CoRoT-10b, $e=0.53 \pm 0.04$, Bonomo et al. 2010; CoRoT-20b, $e=0.562 \pm 0.013$, Deleuil et al. 2012; HD $17156 \mathrm{~b}, e=0.669 \pm 0.008$, Madhusudhan \& Winn 2009; and HD 80606b, $e=0.9330 \pm 0.0005$, Hébrard et al. 2010), all of which have longer orbital periods than HAT-P-34b. Of these planets, HAT-P-2b is most similar in orbital period to HAT-P$34 \mathrm{~b}$, but it has a mass that is more than two times larger than that of HAT-P-34b. Two planets with masses, radii, and equilibrium temperatures within $10 \%$ of the values of HAT-P-34b (assuming zero albedo and full heat redistribution) are CoRoT18b (Hébrard et al. 2011) and WASP-32b (Maxted et al. 2010); however, neither of these planets has a significant eccentricity.

HAT-P-34b is a promising target for measuring the RM effect (Rossiter 1924; McLaughlin 1924), since the host star is bright $(V=10.16)$, has a significant $\operatorname{spin}\left(v \sin i=24.0 \pm 0.5 \mathrm{~km} \mathrm{~s}^{-1}\right)$, and the transit is moderately long $\left(T_{14}=0.1455 \pm 0.0016\right.$ days $)$. Also, the transit is far from equatorial $\left(b=0.336_{-0.128}^{+0.099}\right)$, a configuration that is important for resolving the degeneracy between $v \sin i$ and $\lambda$, which is the sky-plane projected angle between the planetary orbital normal and the stellar spin axis. Winn et al. (2010) pointed out that hot Jupiters around stars with $T_{\text {eff }} \gtrsim 6250 \mathrm{~K}$ have a higher chance of being misaligned. Based on the effective temperature of the host star $6442 \pm 88 \mathrm{~K}$, we thus expect that HAT-P-34b has a higher chance of misalignment (note that this may not necessarily yield a non-zero $\lambda$, if HATP-34b's orbit is tilted along the line of sight). Alternatively, Schlaufman (2010) used a stellar rotation model and observed $v \sin i$ values to statistically identify TEP systems that may be misaligned along the line of sight, and concluded these preferentially occur at $M_{\star}>1.2 M_{\odot}$. Based on the stellar mass alone $\left(1.39 M_{\odot}\right)$ we conclude that the chances for misalignment are increased.

\subsection{HAT-P-35b}

HAT-P-35b is a very typical $M_{p}=1.054 \pm 0.033 M_{\mathrm{J}}, R_{p}=$ $1.332 \pm 0.098 R_{\mathrm{J}}$ planet on a $P=3.646706 \pm 0.000021$ day orbit and with an equilibrium temperature of $T_{\mathrm{eq}}=1581 \pm 45 \mathrm{~K}$ (again, assuming zero albedo and full heat redistribution). There are four other planets with masses, radii, and equilibrium temperatures that are all within $10 \%$ of the values for HATP-35b. These are HAT-P-5b (Bakos et al. 2007b), HAT-P-6b (Noyes et al. 2008), OGLE-TR-211b (Udalski et al. 2008), and WASP-26b (Smalley et al. 2010). The stellar effective temperature $(6096 \pm 88 \mathrm{~K})$ is close to the assumed borderline between well-aligned and misaligned systems, making it an interesting system for testing the RM effect (with the caveat that $v \sin i$, and thus the expected amplitude of the anomaly is low). 

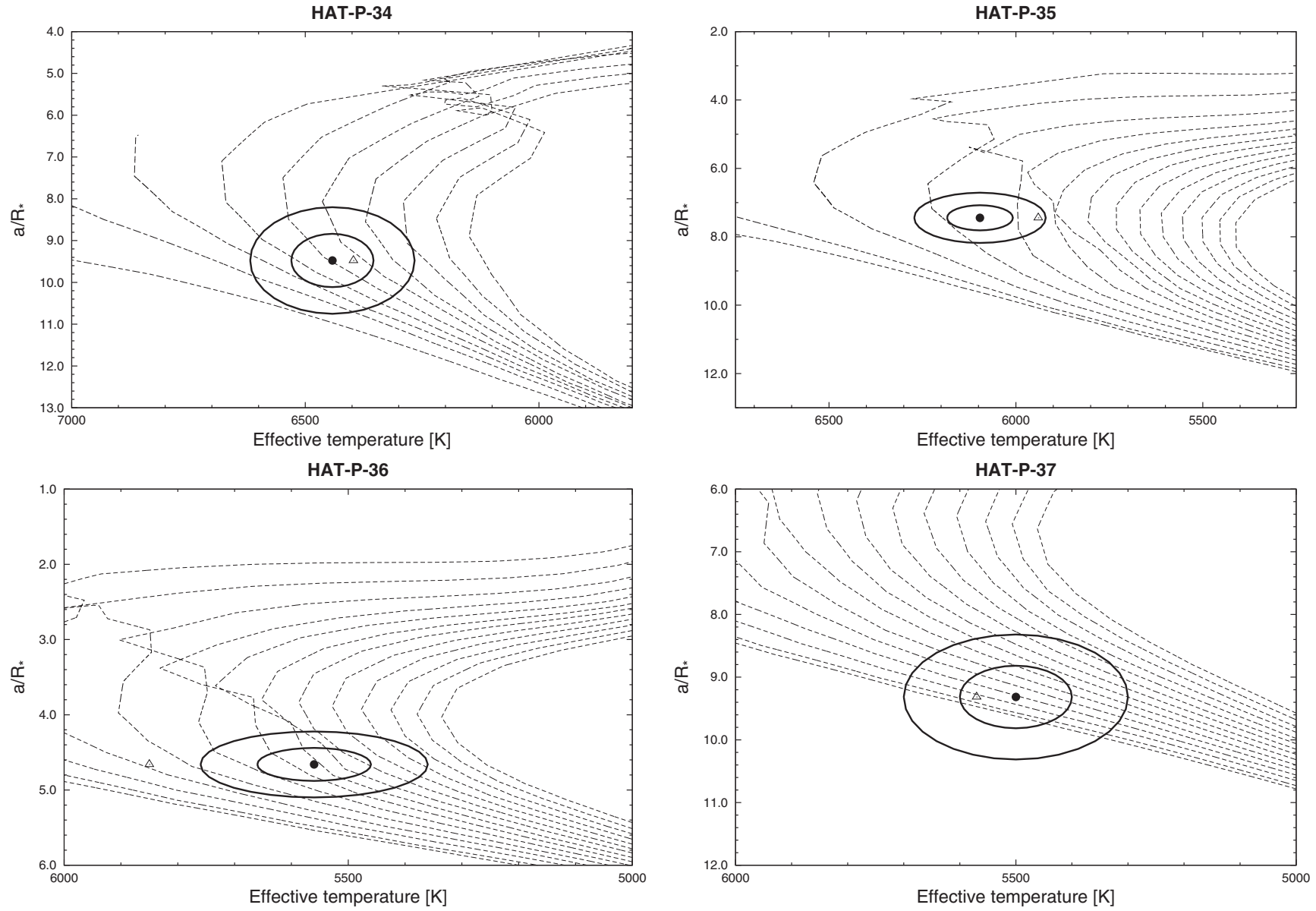

Figure 10. Comparison of the measured values of $T_{\text {eff } \star}$ and $a / R_{\star}$ for HAT-P-34 (upper left), HAT-P-35 (upper right), HAT-P-36 (lower left), and HAT-P-37 (lower right) to model isochrones from Yi et al. (2001). The isochrones are generated for the measured metallicity of each star, and for ages of $0.5 \mathrm{Gyr}$ and $1-3 \mathrm{Gyr}$ in steps of 0.25 Gyr for HAT-P-34 and of 0.5 Gyr and 1-14 Gyr in steps of 1 Gyr for HAT-P-35, HAT-P-36, and HAT-P-37 (ages increase from left to right in each plot). The lines show the $1 \sigma$ and $2 \sigma$ confidence ellipses for the measured parameters. The initial values of $T_{\text {eff } \star}$ and $a / R_{\star}$ from the initial spectroscopic and light-curve analyses are represented with a triangle in each panel.

\subsection{HAT-P-36b}

HAT-P-36b is a very short-period $(P=1.327347 \pm$ 0.000003 days) planet with a mass of $M_{p}=1.832 \pm 0.099 M_{\mathrm{J}}$, a radius of $R_{p}=1.264 \pm 0.071 R_{\mathrm{J}}$, and an equilibrium temperature of $T_{\text {eq }}=1823 \pm 55 \mathrm{~K}$. There are two other planets with masses, radii, and equilibrium temperatures within $10 \%$ of the values for HAT-P-36b: TrES-3b (O'Donovan et al. 2007) and WASP-3b (Pollacco et al. 2008).

\subsection{HAT-P-37b}

Like the preceding planets, HAT-P-37b also has very typical physical properties, with $M_{p}=1.169 \pm 0.103 M_{\mathrm{J}}, R_{p}=$ $1.178 \pm 0.077 R_{\mathrm{J}}, P=2.797436 \pm 0.000007$ days, and $T_{\mathrm{eq}}=$ $1271 \pm 47 \mathrm{~K}$. Three planets with masses, radii, and equilibrium temperatures within $10 \%$ of the values for HAT-P-37b are HD 189733b (Bouchy et al. 2005), OGLE-TR-113b (Bouchy et al. 2004), and XO-5b (Burke et al. 2008). HAT-P-37 lies just outside of the field of view of the Kepler space mission and is listed in the Kepler Input Catalog (KIC) ${ }^{14}$ as KIC 12396036.

\subsection{On the Eccentricity of HAT-P-34b}

According to Adams \& Laughlin (2006), the eccentricity of a hot Jupiter's orbit decays due to both the tides on the

\footnotetext{
14 http://www.cfa.harvard.edu/kepler/kic/kicindex.html
}

star and the tides on the planet, with the tides on the planet dominating the circularization as long as the tidal quality factor of the planet $\left(Q_{P}\right)$ is not much larger than the star's $\left(Q_{\star}\right)$. Both of these factors are highly uncertain with various theoretical and observational constraints ranging over several orders of magnitude. In particular, tidal circularization of mainsequence stars (Claret \& Cunha 1997; Meibom \& Mathieu 2005; Zahn \& Bouchet 1989; Zahn 1989) seems to indicate $10^{5} \lesssim Q_{\star} \lesssim 10^{6}$. On the other hand, the discovery of extremely short-period massive planets, the two most dramatic being WASP-18b (Hellier et al. 2009) and WASP-19b (Hellier et al. 2011), seems to be inconsistent with such efficient dissipation (Penev et al. 2012), requiring much larger values of $Q_{\star} \gtrsim 10^{8}$, which coincide well with the theoretical values derived by Penev \& Sasselov (2011), who argue that binary stars and star-planet systems are subject to different modes of dissipation in the star. The tidal dissipation parameter in the planet has also been the subject of many studies attempting to constrain it either from theory (Bodenheimer et al. 2003; Ogilvie \& Lin 2004) or from the observed configuration of Jupiter's satellites (Goldreich \& Soter 1966) giving $10^{5} \lesssim Q_{P} \lesssim 10^{7}$.

With this in mind we conclude that the circularization of HAT-P-34b's orbit is likely dominated by the tidal dissipation in the planet and using $Q_{P}=10^{6}$ and the expression for the tidal circularization timescale from Adams \& Laughlin (2006), we estimate the eccentricity of HAT-P-34b should decay on the scale 
Table 6

Orbital and Planetary Parameters for HAT-P-34b through HAT-P-37b

\begin{tabular}{|c|c|c|c|c|}
\hline Parameter & $\begin{array}{c}\text { HAT-P-34b } \\
\text { Value }\end{array}$ & $\begin{array}{c}\text { HAT-P-35b } \\
\text { Value }\end{array}$ & $\begin{array}{c}\text { HAT-P-36b } \\
\text { Value }\end{array}$ & $\begin{array}{c}\text { HAT-P-37b } \\
\text { Value }\end{array}$ \\
\hline \multicolumn{5}{|l|}{ Light-curve parameters } \\
\hline$P$ (days) ... & $5.452654 \pm 0.000016$ & $3.646706 \pm 0.000021$ & $1.327347 \pm 0.000003$ & $2.797436 \pm 0.000007$ \\
\hline$T_{c}(\mathrm{BJD})^{\mathrm{a}} \ldots$ & $2455431.59629 \pm 0.00055$ & $2455578.66081 \pm 0.00050$ & $2455565.18144 \pm 0.00020$ & $2455642.14318 \pm 0.00029$ \\
\hline$T_{14}$ (days) $^{\mathrm{a}} \ldots$ & $0.1455 \pm 0.0016$ & $0.1640 \pm 0.0018$ & $0.0923 \pm 0.0007$ & $0.0971 \pm 0.0015$ \\
\hline$T_{12}=T_{34}(\text { days })^{\mathrm{a}} \ldots$ & $0.0121 \pm 0.0013$ & $0.0162 \pm 0.0017$ & $0.0107 \pm 0.0007$ & $0.0153 \pm 0.0013$ \\
\hline$a / R_{\star} \ldots$ & $9.48 \pm 0.64$ & $7.45 \pm 0.37$ & $4.66 \pm 0.22$ & $9.32_{-0.57}^{+0.42}$ \\
\hline$\zeta / R_{\star} \cdots$ & $14.99 \pm 0.09$ & $13.52 \pm 0.09$ & $24.51 \pm 0.14$ & $24.33 \pm 0.18$ \\
\hline$R_{p} / R_{\star} \ldots$ & $0.0801 \pm 0.0026$ & $0.0954 \pm 0.0027$ & $0.1186 \pm 0.0012$ & $0.1378 \pm 0.0030$ \\
\hline$b^{2} \ldots$ & $0.113_{-0.062}^{+0.080}$ & $0.128_{-0.066}^{+0.078}$ & $0.097_{-0.048}^{+0.057}$ & $0.255_{-0.056}^{+0.044}$ \\
\hline$b \equiv a \cos i / R_{\star} \ldots$ & $0.336_{-0.128}^{+0.099}$ & $0.357_{-0.127}^{+0.092}$ & $0.312_{-0.105}^{+0.078}$ & $0.505_{-0.062}^{+0.041}$ \\
\hline$i(\operatorname{deg}) \ldots$ & $87.1 \pm 1.2$ & $87.3 \pm 1.0$ & $86.0 \pm 1.3$ & $86.9_{-0.5}^{+0.4}$ \\
\hline \multicolumn{5}{|c|}{ Quadratic limb-darkening coefficients ${ }^{\text {b }}$} \\
\hline$c_{1}, i$ (linear term) $\ldots$ & 0.1785 & 0.2198 & 0.3142 & 0.3156 \\
\hline$c_{2}, i$ (quadratic term) $\ldots$ & 0.3825 & 0.3587 & 0.3113 & 0.3032 \\
\hline$c_{1}, z \ldots$ & 0.1269 & $\ldots$ & $\ldots$ & 0.2477 \\
\hline$c_{2}, z \ldots$ & 0.3728 & $\ldots$ & $\ldots$ & 0.3082 \\
\hline \multicolumn{5}{|l|}{$\mathrm{RV}$ parameters } \\
\hline$K\left(\mathrm{~m} \mathrm{~s}^{-1}\right) \ldots$ & $343.1 \pm 21.3$ & $120.7 \pm 2.2$ & $334.7 \pm 14.5$ & $177.7 \pm 14.8$ \\
\hline$e \cos \omega^{\mathrm{c}} \ldots$ & $0.410 \pm 0.031$ & $-0.004 \pm 0.013$ & $-0.002 \pm 0.032$ & $-0.017 \pm 0.039$ \\
\hline$e \sin \omega^{\mathrm{c}} \ldots$ & $0.156 \pm 0.052$ & $-0.017 \pm 0.026$ & $0.051 \pm 0.040$ & $0.007 \pm 0.060$ \\
\hline$e \ldots$ & $0.441 \pm 0.032$ & $0.025 \pm 0.018$ & $0.063 \pm 0.032$ & $0.058 \pm 0.038$ \\
\hline$\omega(\operatorname{deg}) \ldots$ & $20 \pm 14$ & $248 \pm 93$ & $95 \pm 63$ & $164 \pm 84$ \\
\hline$\dot{\gamma}\left(\mathrm{m} \mathrm{s}^{-1} \mathrm{~d}^{-1}\right) \ldots$ & $0.8683 \pm 0.4719$ & $\ldots$ & $\ldots$ & $\ldots$ \\
\hline \multicolumn{5}{|l|}{ RV jitter } \\
\hline Keck/HIRES $\left(\mathrm{m} \mathrm{s}^{-1}\right) \ldots$ & 56.0 & 3.7 & $\ldots$ & $\ldots$ \\
\hline Subaru/HDS $\left(\mathrm{m} \mathrm{s}^{-1}\right) \ldots$ & 32.0 & $\ldots$ & $\ldots$ & $\ldots$ \\
\hline NOT/FIES $\left(\mathrm{m} \mathrm{s}^{-1}\right) \ldots$ & 0.0 & $\ldots$ & $\ldots$ & $\ldots$ \\
\hline FLWO 1.5/TRES $\left(\mathrm{ms}^{-1}\right) \ldots$ & $\ldots$ & $\ldots$ & 33.6 & 25.8 \\
\hline \multicolumn{5}{|l|}{ Secondary eclipse parameters } \\
\hline$T_{s}(\mathrm{BJD}) \ldots$ & $2455435.721 \pm 0.099$ & $2455580.476 \pm 0.030$ & $2455565.844 \pm 0.027$ & $2455643.512 \pm 0.070$ \\
\hline$T_{s, 14} \ldots$ & $0.1871 \pm 0.0170$ & $0.1596 \pm 0.0076$ & $0.1013 \pm 0.0071$ & $0.0981 \pm 0.0083$ \\
\hline$T_{s, 12 \ldots}$ & $0.0176 \pm 0.0052$ & $0.0156 \pm 0.0019$ & $0.0120 \pm 0.0015$ & $0.0153 \pm 0.0029$ \\
\hline \multicolumn{5}{|l|}{ Planetary parameters } \\
\hline$M_{p}\left(M_{\mathrm{J}}\right) \ldots$ & $3.328 \pm 0.211$ & $1.054 \pm 0.033$ & $1.832 \pm 0.099$ & $1.169 \pm 0.103$ \\
\hline$R_{p}\left(R_{\mathrm{J}}\right) \ldots$ & $1.197_{-0.092}^{+0.128}$ & $1.332 \pm 0.098$ & $1.264 \pm 0.071$ & $1.178 \pm 0.077$ \\
\hline$C\left(M_{p}, R_{p}\right)^{\mathrm{d}} \ldots$ & 0.23 & 0.49 & 0.11 & 0.02 \\
\hline$\rho_{p}\left(\mathrm{~g} \mathrm{~cm}^{-3}\right) \ldots$ & $2.40 \pm 0.63$ & $0.55 \pm 0.11$ & $1.12 \pm 0.19$ & $0.89 \pm 0.19$ \\
\hline $\log g_{p}(\mathrm{cgs}) \ldots$ & $3.76 \pm 0.08$ & $3.17 \pm 0.06$ & $3.45 \pm 0.05$ & $3.32 \pm 0.07$ \\
\hline$a(\mathrm{AU}) \ldots$ & $0.0677 \pm 0.0008$ & $0.0498 \pm 0.0006$ & $0.0238 \pm 0.0004$ & $0.0379 \pm 0.0006$ \\
\hline$T_{\mathrm{eq}}(\mathrm{K}) \ldots$ & $1520 \pm 60$ & $1581 \pm 45$ & $1823 \pm 55$ & $1271 \pm 47$ \\
\hline$\Theta^{\mathrm{e}} \ldots$ & $0.269 \pm 0.029$ & $0.064 \pm 0.005$ & $0.067 \pm 0.005$ & $0.081 \pm 0.009$ \\
\hline$\langle F\rangle\left(10^{9} \mathrm{erg} \mathrm{s}^{-1} \mathrm{~cm}^{-2}\right)^{\mathrm{f}} \ldots$ & $1.21_{-0.16}^{+0.23}$ & $1.41_{-0.14}^{+0.19}$ & $2.49 \pm 0.30$ & $0.589_{-0.075}^{+0.102}$ \\
\hline
\end{tabular}

Notes.

a $T_{c}$ : Reference epoch of mid-transit that minimizes the correlation with the orbital period. $T_{14}$ : total transit duration, time between first to last contact; $T_{12}=T_{34}$ : ingress/egress time, time between first and second, or third and fourth contact. Barycentric Julian dates (BJD) throughout the paper are calculated from Coordinated Universal Time (UTC).

b Values for a quadratic law, adopted from the tabulations by Claret (2004) according to the spectroscopic (SME) parameters listed in Table 5.

${ }^{\mathrm{c}}$ Lagrangian orbital parameters derived from the global modeling, and primarily determined by the RV data.

${ }^{\mathrm{d}}$ Correlation coefficient between the planetary mass $M_{p}$ and radius $R_{p}$.

e The Safronov number is given by $\Theta=(1 / 2)\left(V_{\text {esc }} / V_{\text {orb }}\right)^{2}=\left(a / R_{p}\right)\left(M_{p} / M_{\star}\right)$ (see Hansen $\&$ Barman 2007).

${ }^{\mathrm{f}}$ Incoming flux per unit surface area, averaged over the orbit.

of $2 \mathrm{Gyr}$, i.e., it is not in conflict with theoretical expectations. The possible outer companion indicated by the RV trend may also be responsible for pumping the eccentricity of the inner planet HAT-P-34b (see Correia et al. 2012 for a discussion).

Figure 11 shows HAT-P-34b on the orbital-period-eccentricity plane of TEPs with well determined parameters (using our own compilation that attempts to keep up with various refinements to these parameters). It is apparent that eccentricity is correlated with orbital period and with planet mass, as expected from tidal theory. HAT-P-34b lies in a sparse position in these diagrams; for example, it has a high eccentricity for its period, the only similar planet being HAT-P-2b.

Figure 12 is a "tidal" plot (see Figure 3 of Pont et al. 2011), showing TEPs with well measured properties in the $a / R_{p}-M_{p} / M_{\star}$ plane, using more data points (including the present discoveries) than Pont et al. (2011). Since $\tau_{c}=$ $(4 / 63) Q_{P} \sqrt{\left(a^{3} / G M_{\star}\right.}\left(a / R_{P}\right)^{5} M_{p} / M_{\star}$, we expect planets with small relative semimajor axis $\left(a / R_{P}\right)$ or planets with small 


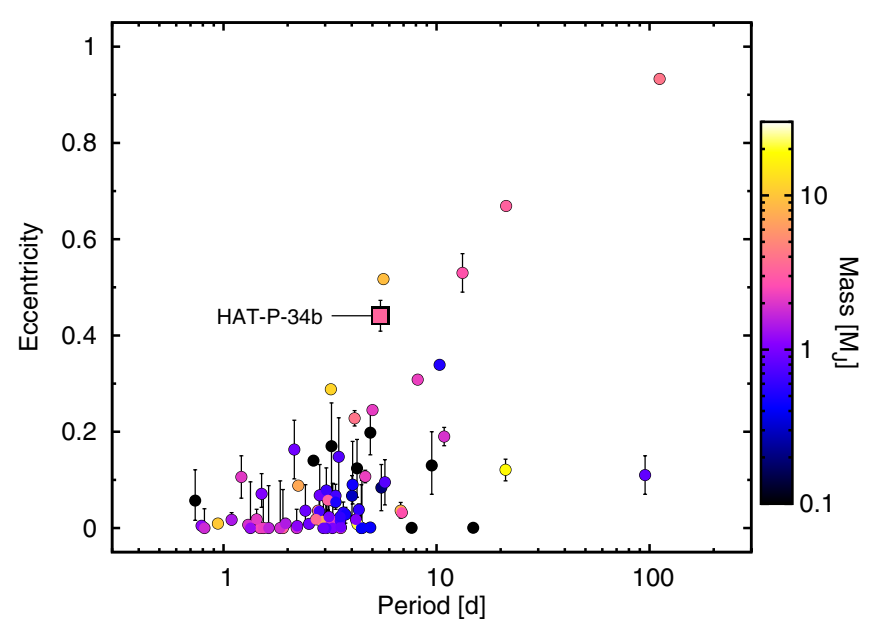

Figure 11. Orbital-period-eccentricity diagram of TEPs with eccentricity uncertainty less than 0.1 . The color (gray-scale shade) of the symbols indicates the mass of each planet. HAT-P-34b is labeled. As expected from tidal evolution theory, high eccentricity planets tend to have longer orbital periods and greater masses.

(A color version of this figure is available in the online journal.)

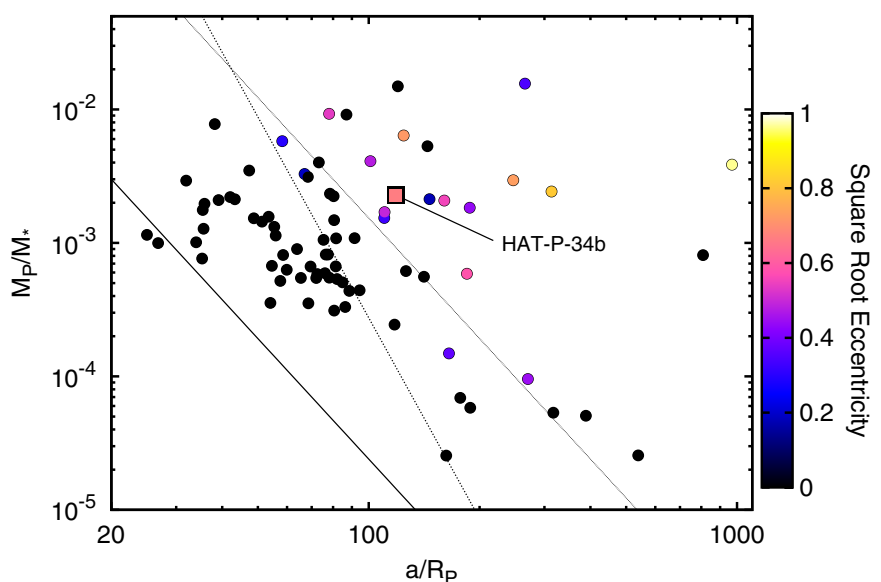

Figure 12. "Tidal" diagram following Figure 3 of Pont et al. (2011). The color (gray-scale shade) of the symbols indicates the eccentricity, we assume zero eccentricity for planets that have a measured eccentricity within $4 \sigma$ of zero. The dotted line shows the locus of points with a circularization timescale of $1 \mathrm{Gyr}$ assuming small eccentricity, $Q_{P}=10^{6}$, and $P=3 \mathrm{~d}$. The thick solid line shows the relation $a=2 a_{H}$ where $a_{H}$ is the semimajor axis at which the radius of the planet equals its Hill radius. The thin solid line shows $a=4 a_{H}$.

(A color version of this figure is available in the online journal.)

relative mass $\left(M_{p} / M_{\star}\right)$ to be circularized. This is indeed the case, as shown by the intensity (color) scale representing eccentricity. For hot Jupiters that migrate in by circularization of an initially very eccentric orbit, the expected "parking distance" is $\sim 2 a_{H}$ (Ford \& Rasio 2006), where $a_{H}$ is the semimajor axis at which the radius of the planet equals its Hill radius. The thick solid line in Figure 12 shows this relation. A fairly good match for the dividing line between the circularized (denoted by black points) and eccentric (gray or color) points is at $a \approx 4 a_{H}$ (marked with a thin solid line). This relation now includes very small mass Kepler discoveries. HAT-P-34b belongs to the sparse group of high relative semimajor axis $\left(a / R_{p}\right)$ and massive extrasolar planets.

HATNet operations have been funded by NASA grants NNG04GN74G and NNX08AF23G. We acknowledge partial funding of the HATNet follow-up effort from NSF AST-
1108686. We acknowledge partial support also from the Kepler Mission under NASA Cooperative Agreement NCC2-1390 (D.W.L. and PI). G.K. thanks the Hungarian Scientific Research Foundation (OTKA) for support through grant K-81373. This research has made use of Keck telescope time granted through NOAO (program A289Hr) and NASA (N167Hr and N029Hr). This paper uses observations obtained with facilities of the Las Cumbres Observatory Global Telescope. Data presented in this paper are based on observations obtained at the HAT station at the Submillimeter Array of SAO, and HAT station at the Fred Lawrence Whipple Observatory of SAO. The authors recognize and acknowledge the very significant cultural role and reverence that the summit of Mauna Kea has always had within the indigenous Hawaiian community. We are most fortunate to have the opportunity to conduct observations from this mountain.

\section{APPENDIX}

\section{SPECTROSCOPIC AND PHOTOMETRIC DATA}

The following tables present the spectroscopic data (radial velocities, bisector spans, and activity index measurements)

Table 7

Relative Radial Velocities, Bisector Spans, and Activity Index Measurements of HAT-P-34

\begin{tabular}{lrrccccc}
\hline \hline BJD & $\begin{array}{c}\mathrm{RV}^{\mathrm{a}} \\
\left(\mathrm{m} \mathrm{s}^{-1}\right)\end{array}$ & $\begin{array}{c}\sigma_{\mathrm{RV}}^{\mathrm{b}} \\
\left(\mathrm{m} \mathrm{s}^{-1}\right)\end{array}$ & $\begin{array}{c}\mathrm{BS} \\
\left(\mathrm{m} \mathrm{s}^{-1}\right)\end{array}$ & $\begin{array}{c}\sigma_{\mathrm{BS}} \\
\left(\mathrm{m} \mathrm{s}^{-1}\right)\end{array}$ & $\mathrm{S}^{\mathrm{c}}$ & Phase & Instrument \\
\hline 1339.92722 & -158.62 & 50.92 & $\ldots$ & $\ldots$ & $\ldots$ & 0.188 & Subaru \\
1339.93516 & -201.37 & 57.36 & $\ldots$ & $\ldots$ & $\ldots$ & 0.190 & Subaru \\
1339.94290 & -204.06 & 44.53 & $\ldots$ & $\ldots$ & $\ldots$ & 0.191 & Subaru \\
1341.11630 & -246.80 & 47.11 & $\ldots$ & $\ldots$ & $\ldots$ & 0.406 & Subaru \\
1341.12058 & -204.87 & 47.32 & $\ldots$ & $\ldots$ & $\ldots$ & 0.407 & Subaru \\
1341.12485 & -193.24 & 43.00 & $\ldots$ & $\ldots$ & $\ldots$ & 0.408 & Subaru \\
1374.12069 & -196.46 & 11.37 & -2.85 & 6.57 & 0.176 & 0.459 & Keck \\
1374.85735 & $\ldots$ & $\ldots$ & -9.20 & 4.18 & 0.173 & 0.594 & Keck \\
1374.86800 & -80.49 & 11.89 & -25.52 & 9.34 & 0.176 & 0.596 & Keck \\
1375.95203 & 285.94 & 10.24 & -15.73 & 4.46 & 0.178 & 0.795 & Keck \\
1378.12823 & -265.18 & 12.71 & -13.52 & 7.86 & 0.174 & 0.194 & Keck \\
1379.07458 & -226.26 & 12.73 & -10.85 & 3.72 & 0.176 & 0.368 & Keck \\
1379.56536 & -162.68 & 61.60 & $\ldots$ & $\ldots$ & $\ldots$ & 0.458 & FIES \\
1380.13344 & -135.85 & 13.51 & 60.87 & 20.44 & 0.180 & 0.562 & Keck \\
1380.49378 & 3.45 & 58.70 & $\ldots$ & $\ldots$ & $\ldots$ & 0.628 & FIES \\
1381.11125 & 110.87 & 14.71 & -9.04 & 12.64 & 0.175 & 0.741 & Keck \\
1381.55600 & 269.57 & 46.50 & $\ldots$ & $\ldots$ & $\ldots$ & 0.823 & FIES \\
1383.47949 & -108.72 & 56.00 & $\ldots$ & $\ldots$ & $\ldots$ & 0.176 & FIES \\
1384.48968 & -173.76 & 50.60 & $\ldots$ & $\ldots$ & $\ldots$ & 0.361 & FIES \\
1400.85414 & -141.78 & 15.43 & 27.38 & 7.53 & 0.167 & 0.362 & Keck \\
1403.82567 & 497.16 & 15.18 & 38.35 & 6.14 & 0.173 & 0.907 & Keck \\
1404.83902 & 4.37 & 14.05 & 0.79 & 7.25 & 0.174 & 0.093 & Keck \\
1415.04513 & 310.06 & 14.03 & 2.76 & 8.29 & 0.168 & 0.965 & Keck \\
1423.66787 & -115.78 & 78.50 & $\ldots$ & $\ldots$ & $\ldots$ & 0.546 & FIES \\
1424.64318 & 41.31 & 139.40 & $\ldots$ & $\ldots$ & $\ldots$ & 0.725 & FIES \\
1425.57489 & 474.54 & 48.70 & $\ldots$ & $\ldots$ & $\ldots$ & 0.896 & FIES \\
1426.64513 & -154.35 & 57.60 & $\ldots$ & $\ldots$ & $\ldots$ & 0.092 & FIES \\
1464.85484 & -99.38 & 15.03 & -11.41 & 11.10 & 0.164 & 0.100 & Keck \\
1465.94874 & -249.34 & 13.70 & -19.33 & 6.92 & 0.163 & 0.300 & Keck \\
\hline & 0.98 & 13.61 & -12.70 & 8.00 & 0.164 & 0.625 & Keck \\
\hline & & & & & & &
\end{tabular}

Notes. Note that for the iodine-free template exposures we do not measure the RV but do measure the BS and S index. Such template exposures can be distinguished by the missing RV value.

a The zero point of these velocities is arbitrary. An overall offset $\gamma_{\text {rel }}$ fitted to these velocities in Section 3.2 has not been subtracted.

$\mathrm{b}$ Internal errors excluding the component of astrophysical jitter considered in Section 3.2.

${ }^{\mathrm{c}}$ Chromospheric activity index, calibrated to the scale of Vaughan et al. (1978). 
Table 8

Relative Radial Velocities, Bisector Spans, and Activity Index Measurements of HAT-P-35

\begin{tabular}{lccrcccc}
\hline \hline $\begin{array}{l}\text { BJD } \\
(2,454,000+)\end{array}$ & $\begin{array}{c}\mathrm{RV}^{\mathrm{a}} \\
\left(\mathrm{m} \mathrm{s}^{-1}\right)\end{array}$ & $\begin{array}{c}\sigma_{\mathrm{RV}}^{\mathrm{b}} \\
\left(\mathrm{m} \mathrm{s}^{-1}\right)\end{array}$ & $\begin{array}{c}\mathrm{BS} \\
\left(\mathrm{m} \mathrm{s}^{-1}\right)\end{array}$ & $\begin{array}{c}\sigma_{\mathrm{BS}} \\
\left(\mathrm{m} \mathrm{s}^{-1}\right)\end{array}$ & $\mathrm{S}^{\mathrm{c}}$ & Phase & Instrument \\
\hline 1466.12094 & -92.77 & 2.40 & -7.06 & 3.64 & 0.129 & 0.139 & Keck \\
1468.11371 & 111.39 & 1.86 & 4.37 & 2.92 & 0.129 & 0.686 & Keck \\
1468.12932 & $\ldots$ & $\ldots$ & 0.45 & 2.76 & 0.129 & 0.690 & Keck \\
1470.12970 & -118.71 & 2.72 & 2.26 & 2.07 & 0.124 & 0.239 & Keck \\
$1482.73469^{\mathrm{d}}$ & 106.37 & 10.20 & 7.70 & 9.60 & $\ldots$ & 0.695 & FIES \\
$1483.74899^{\mathrm{d}}$ & 39.07 & 10.70 & -17.40 & 6.50 & $\ldots$ & 0.973 & FIES \\
$1486.74544^{\mathrm{d}}$ & 97.37 & 8.50 & 8.90 & 8.30 & $\ldots$ & 0.795 & FIES \\
$1488.74737^{\mathrm{d}}$ & -152.93 & 16.90 & 24.70 & 17.20 & $\ldots$ & 0.344 & FIES \\
$1490.72460^{\mathrm{d}}$ & 78.27 & 8.50 & -23.80 & 6.40 & $\ldots$ & 0.886 & FIES \\
1501.03468 & 117.01 & 2.39 & 9.98 & 2.63 & 0.127 & 0.713 & Keck \\
1523.09695 & 126.39 & 2.09 & 0.65 & 4.88 & 0.134 & 0.763 & Keck \\
1529.15460 & -55.78 & 2.32 & -11.07 & 2.00 & 0.129 & 0.424 & Keck \\
1545.14866 & 103.29 & 3.12 & 9.13 & 3.56 & 0.125 & 0.810 & Keck \\
\hline
\end{tabular}

Notes. Note that for the iodine-free template exposures we do not measure the RV but do measure the BS and S index. Such template exposures can be distinguished by the missing RV value.

a The zero point of these velocities is arbitrary. An overall offset $\gamma_{\text {rel }}$ fitted to these velocities in Section 3.2 has not been subtracted.

b Internal errors excluding the component of astrophysical jitter considered in Section 3.2.

${ }^{c}$ Chromospheric activity index, calibrated to the scale of Vaughan et al. (1978).

${ }^{d}$ The FIES/NOT observations of HAT-P-35 were not used in the analysis, see the footnote to Table 4 . Transit ingress began during the hour-long exposure obtained at phase 0.973 , and the exposure obtained at phase 0.344 has a low $\mathrm{S} / \mathrm{N}$ ratio and was obtained during morning twilight.

Table 9

Relative Radial Velocities, Bisector Spans, and Activity Index Measurements of HAT-P-36

\begin{tabular}{lrrrrr}
\hline \hline $\begin{array}{l}\text { BJD } \\
(2,454,000+)\end{array}$ & $\begin{array}{c}\mathrm{RV}^{\mathrm{a}} \\
\left(\mathrm{m} \mathrm{s}^{-1}\right)\end{array}$ & $\begin{array}{c}\sigma_{\mathrm{RV}}^{\mathrm{b}} \\
\left(\mathrm{m} \mathrm{s}^{-1}\right)\end{array}$ & $\begin{array}{c}\mathrm{BS} \\
\left(\mathrm{m} \mathrm{s}^{-1}\right)\end{array}$ & $\begin{array}{c}\sigma_{\mathrm{BS}} \\
\left(\mathrm{m} \mathrm{s}^{-1}\right)\end{array}$ & Phase \\
\hline 1547.02139 & -206.37 & 22.40 & 20.50 & 15.80 & 0.319 \\
1550.04236 & 200.83 & 22.80 & -11.40 & 21.60 & 0.594 \\
1554.99394 & -293.87 & 11.40 & -7.50 & 7.50 & 0.325 \\
1556.05388 & -237.67 & 7.40 & 3.20 & 7.70 & 0.123 \\
1557.05533 & 240.83 & 24.60 & 5.10 & 18.40 & 0.878 \\
1558.95741 & -355.47 & 10.50 & 4.30 & 8.70 & 0.311 \\
1559.99613 & -199.97 & 17.70 & -14.10 & 17.40 & 0.093 \\
1576.04204 & -326.97 & 16.70 & $\ldots$ & $\cdots$ & 0.182 \\
1576.91790 & 331.53 & 14.40 & $\ldots$ & $\cdots$ & 0.842 \\
1578.04243 & 265.83 & 7.40 & $\cdots$ & $\cdots$ & 0.689 \\
1579.96119 & -277.57 & 9.00 & $\cdots$ & $\cdots$ & 0.135 \\
1580.92488 & 257.33 & 12.40 & $\cdots$ & $\cdots$ & 0.861 \\
\hline
\end{tabular}

Notes.

${ }^{\text {a }}$ The zero point of these velocities is arbitrary. An overall offset $\gamma_{\text {rel }}$ fitted to these velocities in Section 3.2 has not been subtracted.

${ }^{b}$ Internal errors excluding the component of astrophysical jitter considered in Section 3.2.

and high-precision photometric data for the four planets presented in this paper. Measurements derived from the highprecision spectroscopic observations of HAT-P-34 through HAT-P-37 are given in Tables 7-10, respectively. Tables 11-14 give the follow-up photometric observations for each of these systems.
Table 10

Relative Radial Velocities, Bisector Spans, and Activity Index Measurements of HAT-P-37

\begin{tabular}{lrcrcr}
\hline \hline $\begin{array}{l}\text { BJD } \\
(2,454,000+)\end{array}$ & $\begin{array}{c}\mathrm{RV}^{\mathrm{a}} \\
\left(\mathrm{m} \mathrm{s}^{-1}\right)\end{array}$ & $\begin{array}{c}\sigma_{\mathrm{RV}}{ }^{\mathrm{b}} \\
\left(\mathrm{m} \mathrm{s}^{-1}\right)\end{array}$ & $\begin{array}{c}\mathrm{BS} \\
\left(\mathrm{m} \mathrm{s}^{-1}\right)\end{array}$ & $\begin{array}{c}\sigma_{\mathrm{BS}} \\
\left(\mathrm{m} \mathrm{s}^{-1}\right)\end{array}$ & Phase \\
\hline 1646.92799 & 146.43 & 35.50 & 26.20 & 16.30 & 0.710 \\
1648.01278 & -111.17 & 24.60 & -28.00 & 16.30 & 0.098 \\
1648.91342 & -88.77 & 23.20 & 0.60 & 22.20 & 0.420 \\
1649.89870 & 172.53 & 29.40 & 8.30 & 24.10 & 0.772 \\
1650.96241 & -102.97 & 17.50 & 12.30 & 16.90 & 0.153 \\
1652.92638 & 128.33 & 26.30 & -13.70 & 15.00 & 0.855 \\
1656.98738 & -238.57 & 12.80 & -28.70 & 23.90 & 0.306 \\
1658.96338 & -19.87 & 20.90 & -9.00 & 17.00 & 0.013 \\
1663.90569 & 169.23 & 20.60 & -5.60 & 15.10 & 0.779 \\
1664.91140 & -163.77 & 25.40 & -28.30 & 19.50 & 0.139 \\
1665.91961 & 62.93 & 32.60 & -29.50 & 16.80 & 0.499 \\
1671.84933 & 125.23 & 35.30 & 47.40 & 27.20 & 0.619 \\
1698.79464 & -143.97 & 25.00 & 48.00 & 16.20 & 0.251 \\
\hline
\end{tabular}

Notes.

${ }^{a}$ The zero point of these velocities is arbitrary. An overall offset $\gamma_{\text {rel }}$ fitted to these velocities in Section 3.2 has not been subtracted.

b Internal errors excluding the component of astrophysical jitter considered in Section 3.2.

Table 11

High-precision Differential Photometry of HAT-P-34

\begin{tabular}{lrccc}
\hline \hline $\begin{array}{l}\text { BJD } \\
(2,400,000+)\end{array}$ & \multicolumn{1}{c}{ Mag $^{\mathrm{a}}$} & $\sigma_{\text {Mag }}$ & Mag(orig) $^{\mathrm{b}}$ & Filter \\
\hline 55338.79359 & 0.00011 & 0.00080 & 10.12720 & $i$ \\
55338.79427 & -0.00108 & 0.00080 & 10.12640 & $i$ \\
55338.79494 & 0.00263 & 0.00080 & 10.12910 & $i$ \\
55338.79563 & -0.00041 & 0.00080 & 10.12660 & $i$ \\
55338.79633 & 0.00158 & 0.00080 & 10.12850 & $i$ \\
55338.79701 & 0.00036 & 0.00079 & 10.12720 & $i$ \\
55338.79769 & -0.00243 & 0.00079 & 10.12440 & $i$ \\
55338.79837 & -0.00305 & 0.00079 & 10.12340 & $i$ \\
55338.79905 & 0.00184 & 0.00079 & 10.12940 & $i$ \\
55338.79969 & 0.00187 & 0.00079 & 10.12900 & $i$ \\
\hline
\end{tabular}

Notes.

a The out-of-transit level has been subtracted. These magnitudes have been subjected to the EPD and TFA procedures, carried out simultaneously with the transit fit.

${ }^{\mathrm{b}}$ Raw magnitude values without application of the EPD and TFA procedures.

(This table is available in its entirety in machine-readable and Virtual Observatory (VO) forms in the online journal. A portion is shown here for guidance regarding its form and content.)

Table 12

High-precision Differential Photometry of HAT-P-35

\begin{tabular}{lcccc}
\hline \hline $\begin{array}{l}\text { BJD } \\
(2,400,000+)\end{array}$ & Mag $^{\mathrm{a}}$ & $\sigma_{\text {Mag }}$ & Mag(orig) & Filter \\
\hline 55578.68612 & 0.00996 & 0.00115 & 11.35390 & $i$ \\
55578.68766 & 0.01222 & 0.00104 & 11.35670 & $i$ \\
55578.68921 & 0.01205 & 0.00101 & 11.35720 & $i$ \\
55578.69075 & 0.00983 & 0.00097 & 11.35550 & $i$ \\
55578.69228 & 0.01193 & 0.00099 & 11.35670 & $i$ \\
55578.69383 & 0.01274 & 0.00113 & 11.35760 & $i$ \\
55578.69504 & 0.01155 & 0.00113 & 11.35620 & $i$ \\
55578.69622 & 0.01243 & 0.00115 & 11.35790 & $i$ \\
55578.69742 & 0.01049 & 0.00114 & 11.35540 & $i$ \\
55578.69863 & 0.00960 & 0.00114 & 11.35430 & $i$ \\
& & & &
\end{tabular}

Notes.

${ }^{a}$ The out-of-transit level has been subtracted. These magnitudes have been subjected to the EPD and TFA procedures, carried out simultaneously with the transit fit.

${ }^{\mathrm{b}}$ Raw magnitude values without application of the EPD and TFA procedures.

(This table is available in its entirety in machine-readable and Virtual Observatory (VO) forms in the online journal. A portion is shown here for guidance regarding its form and content.) 
Table 13

High-precision Differential Photometry of HAT-P-36

\begin{tabular}{lcccr}
\hline \hline $\begin{array}{l}\text { BJD } \\
(2,400,000+)\end{array}$ & Mag $^{\mathrm{a}}$ & $\sigma_{\text {Mag }}$ & Mag(orig) $^{\mathrm{b}}$ & Filter \\
\hline 55555.84870 & 0.00292 & 0.00081 & 10.95490 & $i$ \\
55555.85050 & 0.00957 & 0.00083 & 10.96050 & $i$ \\
55555.85205 & 0.00817 & 0.00078 & 10.96100 & $i$ \\
55555.85362 & 0.01416 & 0.00079 & 10.96370 & $i$ \\
55555.85516 & 0.01203 & 0.00079 & 10.96260 & $i$ \\
55555.85671 & 0.01521 & 0.00079 & 10.96600 & $i$ \\
55555.85826 & 0.01690 & 0.00078 & 10.96760 & $i$ \\
55555.85981 & 0.01833 & 0.00079 & 10.96870 & $i$ \\
55555.86135 & 0.01590 & 0.00078 & 10.96410 & $i$ \\
55555.86292 & 0.01733 & 0.00078 & 10.96540 & $i$ \\
\hline
\end{tabular}

Notes.

a The out-of-transit level has been subtracted. These magnitudes have been subjected to the EPD and TFA procedures, carried out simultaneously with the transit fit.

${ }^{b}$ Raw magnitude values without application of the EPD and TFA procedures.

(This table is available in its entirety in machine-readable and Virtual Observatory (VO) forms in the online journal. A portion is shown here for guidance regarding its form and content.)

Table 14

High-precision Differential Photometry of HAT-P-37

\begin{tabular}{lcccc}
\hline \hline $\begin{array}{l}\text { BJD } \\
(2,400,000+)\end{array}$ & Mag $^{\mathrm{a}}$ & $\sigma_{\text {Mag }}$ & Mag(orig) & Filter \\
\hline 55616.96855 & 0.02527 & 0.00133 & 12.41350 & $i$ \\
55616.97081 & 0.02387 & 0.00135 & 12.41200 & $i$ \\
55616.97308 & 0.02220 & 0.00133 & 12.41020 & $i$ \\
55616.97540 & 0.02311 & 0.00131 & 12.41290 & $i$ \\
55616.97774 & 0.02297 & 0.00132 & 12.41110 & $i$ \\
55616.97986 & 0.02149 & 0.00133 & 12.40940 & $i$ \\
55616.98176 & 0.02186 & 0.00133 & 12.40980 & $i$ \\
55616.98366 & 0.01909 & 0.00131 & 12.40740 & $i$ \\
55616.98555 & 0.02301 & 0.00132 & 12.41180 & $i$ \\
55616.98744 & 0.02165 & 0.00129 & 12.40910 & $i$ \\
\hline
\end{tabular}

Notes.

a The out-of-transit level has been subtracted. These magnitudes have been subjected to the EPD and TFA procedures, carried out simultaneously with the transit fit.

${ }^{\mathrm{b}}$ Raw magnitude values without application of the EPD and TFA procedures.

(This table is available in its entirety in machine-readable and Virtual Observatory (VO) forms in the online journal. A portion is shown here for guidance regarding its form and content.)

\section{REFERENCES}

Adams, F. C., \& Laughlin, G. 2006, ApJ, 649, 1004

Bakos, G. Á., Kovács, G., Torres, G., et al. 2007a, ApJ, 670, 826

Bakos, G. Á., Noyes, R. W., Kovács, G., et al. 2004, PASP, 116, 266

Bakos, G. Á., Shporer, A., Pál, A., et al. 2007b, ApJ, 671, L173

Bakos, G. Á., Torres, G., Pál, A., et al. 2010, ApJ, 710, 1724

Bodenheimer, P., Laughlin, G., \& Lin, D. N. C. 2003, ApJ, 592, 555

Bonomo, A. S., Santerne, A., Alonso, R., et al. 2010, A\&A, 520, A65

Borucki, W. J., Koch, D. G., Basri, G., et al. 2011, ApJ, 736, 19

Bouchy, F., Pont, F., Santos, N. C., et al. 2004, A\&A, 421, L13

Bouchy, F., Udry, S., Mayor, M., et al. 2005, A\&A, 444, L15

Buchhave, L. A., Bakos, G. Á., Hartman, J. D., et al. 2010, ApJ, 720, 1118

Burke, C. J., McCullough, P. R., Valenti, J. A., et al. 2008, ApJ, 686, 1331

Butler, R. P., Marcy, G. W., Williams, E., et al. 1996, PASP, 108, 500

Carpenter, J. M. 2001, AJ, 121, 2851

Cegla, H. M., Watson, C. A., Marsh, T. R., et al. 2012, MNRAS, 421, L54

Claret, A. 2004, A\&A, 428, 1001
Claret, A., \& Cunha, N. C. S. 1997, A\&A, 318, 187

Correia, A. C. M., Boué, G., \& Laskar, J. 2012, ApJ, 744, L23

Deleuil, M., Bonomo, A. S., Ferraz-Mello, S., et al. 2012, A\&A, 538, A145

Djupvik, A. A., \& Andersen, J. 2010, in Highlights of Spanish Astrophysics V, ed. J. M. Diego, L. J. Goicoechea, J. I. González-Serrano, \& J. Gorgas (Springer: Berlin), 211

Droege, T. F., Richmond, M. W., \& Sallman, M. 2006, PASP, 118, 1666

Ford, E. B., \& Rasio, F. A. 2006, ApJ, 638, L45

Fûrész, G. 2008, Ph.D. thesis, Univ. Szeged, Hungary

Goldreich, P., \& Soter, S. 1966, Icarus, 5, 375

Hansen, B. M. S., \& Barman, T. 2007, ApJ, 671, 861

Hartman, J. D., Bakos, G. Á., Kipping, D. M., et al. 2011a, ApJ, 728, 138

Hartman, J. D., Bakos, G. Á., Torres, G., et al. 2009, ApJ, 706, 785

Hartman, J. D., Bakos, G. Á., Torres, G., et al. 2011b, ApJ, 742, 59

Hébrard, G., Désert, J.-M., Díaz, R. F., et al. 2010, A\&A, 516, A95

Hébrard, G., Evans, T. M., \& Alonso, R. 2011, A\&A, 533, A130

Hellier, C., Anderson, D. R., Collier Cameron, A., et al. 2009, Nature, 460, 1098

Hellier, C., Anderson, D. R., Collier Cameron, A., et al. 2011, ApJ, 730, L31

Howard, A. W., Johnson, J. A., Marcy, G. W., et al. 2010, ApJ, 721, 1467

Howard, A. W., Marcy, G. W., Bryson, S. T., et al. 2011, arXiv:1103.2541

Isaacson, H., \& Fischer, D. 2010, ApJ, 725, 875

Johnson, J. A., Winn, J. N., Albrecht, S., et al. 2009, PASP, 121, 1104

Johnson, J. A., Winn, J. N., Bakos, G. Á., et al. 2011, ApJ, 735, 24

Kovács, G., Bakos, G. Á., Hartman, J. D., et al. 2010, ApJ, 724, 866

Kovács, G., Bakos, G. Á., \& Noyes, R. W. 2005, MNRAS, 356, 557

Kovács, G., Zucker, S., \& Mazeh, T. 2002, A\&A, 391, 369

Kurucz, R. L. 2005, Mem. Soc. Astron. Ital. Suppl., 8, 14

Lasker, B. M., Lattanzi, M. G., McLean, B. J., et al. 2008, AJ, 136, 735

Latham, D. W., Bakos, G. Á., Torres, G., et al. 2009, ApJ, 704, 1107

Latham, D. W., Rowe, J. F., Quinn, S. N., et al. 2011, ApJ, 732, L24

Lissauer, J. J., Ragozzine, D., Fabrycky, D. C., et al. 2011, ApJS, 197, 8

Madhusudhan, N., \& Winn, J. N. 2009, ApJ, 693, 784

Makarov, V. V., Beichman, C. A., Catanzarite, J. H., et al. 2009, ApJ, 707, L73

Mandel, K., \& Agol, E. 2002, ApJ, 580, L171

Marcy, G. W., \& Butler, R. P. 1992, PASP, 104, 270

Martínez-Arnáiz, R., Maldonado, J., Montes, D., Eiroa, C., \& Montesinos, B. 2010, A\&A, 520, A79

Maxted, P. F. L., Anderson, D. R., Collier Cameron, A., et al. 2010, PASP, 122, 1465

McLaughlin, D. B. 1924, ApJ, 60, 22

Meibom, S., \& Mathieu, R. D. 2005, ApJ, 620, 970

Noguchi, K., Aoki, W., Kawanomoto, S., et al. 2002, PASJ, 54, 855

Noyes, R. W., Bakos, G. Á., Torres, G., et al. 2008, ApJ, 673, L79

Noyes, R. W., Hartmann, L. W., Baliunas, S. L., Duncan, D. K., \& Vaughan, A. H. 1984, ApJ, 279, 763

O’Donovan, F. T., Charbonneau, D., Bakos, G. Á., et al. 2007, ApJ, 663, L37

Ogilvie, G. I., \& Lin, D. N. C. 2004, ApJ, 610, 477

Pál, A. 2009a, MNRAS, 396, 1737

Pál, A. 2009b, PhD thesis, Depart. Astronomy, Eőtvős Loránd Univ.

Pál, A., Bakos, G. Á., Torres, G., et al. 2010, MNRAS, 401, 2665

Penev, K., Jackson, B., Spada, F., \& Thom, N. 2012, ApJ, 751, 96

Penev, K., \& Sasselov, D. 2011, ApJ, 731, 67

Pollacco, D., Skillen, I., Collier Cameron, A., et al. 2008, MNRAS, 385, 1576

Pont, F., Husnoo, N., Mazeh, T., \& Fabrycky, D. 2011, MNRAS, 414, 1278

Queloz, D., Henry, G. W., Sivan, J. P., et al. 2001, A\&A, 379, 279

Quinn, S. N., Bakos, G. Á., Hartman, J., et al. 2012, ApJ, 750, 80

Rauer, H. 2011, in Detection and Dynamics of Transiting Exoplanets, St. Michel l'Observatoire, France, ed. F. Bouchy, R. Díaz, \& C. Moutou (EPJ Web of Conferences, Vol. 11, 07001; Les Ulis: EDP Sciences)

Rossiter, R. A. 1924, ApJ, 60, 15

Saar, S. H., Hatzes, A., Cochran, W., \& Paulson, D. 2003, in The Future of Cool-Star Astrophysics: 12th Cambridge Workshop on Cool Stars, Stellar Systems, and the Sun, ed. A. Brown, G. M. Harper, \& T. R. Ayres (Boulder, CO: Univ. Colorado), 694

Sato, B., Fischer, D. A., Henry, G. W., et al. 2005, ApJ, 633, 465

Sato, B., Kambe, E., Takeda, Y., Izumiura, H., \& Ando, H. 2002, PASJ, 54, 873

Schlaufman, K. C. 2010, ApJ, 719, 602

Schneider, J., Dedieu, C., Le Sidaner, P., Savalle, R., \& Zolotukhin, I. 2011, A\&A, 532, A79

Skrutskie, M. F., Cutri, R. M., Stiening, R., et al. 2006, AJ, 131, 1163

Smalley, B., Anderson, D. R., Collier Cameron, A., et al. 2010, A\&A, 520, A56

Sozzetti, A., Torres, G., Charbonneau, D., et al. 2007, ApJ, 664, 1190 
Torres, G., Bakos, G. Á., Kovács, G., et al. 2007, ApJ, 666, L121

Udalski, A., Pont, F., Naef, D., et al. 2008, A\&A, 482, 299

Valenti, J. A., \& Fischer, D. A. 2005, ApJS, 159, 141

Valenti, J. A., \& Piskunov, N. 1996, A\&AS, 118, 595

Vaughan, A. H., Preston, G. W., \& Wilson, O. C. 1978, PASP, 90, 267

Vogt, S. S., Allen, S. L., Bigelow, B. C., et al. 1994, Proc. SPIE, 2198, 362
Winn, J. N., Fabrycky, D., Albrecht, S., \& Johnson, J. A. 2010, ApJ, 718, L145 Wright, J. T. 2005, PASP, 117, 657

Wright, J. T., Fakhouri, O., Marcy, G. W., et al. 2011, PASP, 123, 412

Yi, S. K., Demarque, P., Kim, Y.-C., et al. 2001, ApJS, 136, 417

Zahn, J.-P. 1989, A\&A, 220, 112

Zahn, J.-P., \& Bouchet, L. 1989, A\&A, 223, 112 